\title{
Rigorous Estimates for Effective Creep-coefficients of Microcracked Masonry Accounting for Cracks Interactions
}

\author{
Amna Rekik ${ }^{1 *}$ \\ ${ }^{1}$ University of Orléans, Polytech Orléans, Laboratoire de Mécanique Gabriel LaMé, Unité de recherche EA 7494, Site Vinci, 8 Rue \\ Léonard de Vinci, 45072 Orléans, France \\ *Corresponding author, e-mail: amna.rekik@univ-orleans.fr
}

Received: 06 April 2019, Accepted: 16 February 2020, Published online: 16 April 2020

\begin{abstract}
Based on the association of finite elements homogenization method and a rigorous homogenization scheme accounting for crack interactions, this paper provides rigorous predictions for the local and effective properties of microcracked viscoelastic masonry with or without creep of bricks. For the sake of simplicity, viscoelastic brick and mortar are assumed to follow the Generalized Maxwell rheological model and to be respectively safe and microcracked. In the mortar, the distribution of microcracks orientations is assumed to be random. Two steps are followed. The first one is based on the identification at the short and long terms of an approximate analytical creep function for the mortar. This step relies on the coupling between the Griffith's brittle fracture theory and a rigorous homogenization scheme - the Ponte Castañeda \& Willis model - accounting for crack interaction instead of the dilute scheme adopted previously in Rekik et al. Two cases are considered: open and closed cracks. The first step allows to avoid recourse to 'heavy' numerical inversion of the Laplace-Carson transform. The second one provides overall creep coefficients of masonry by means of periodic homogenization carried out by finite elements method. For open cracks state, time-dependent crack density is investigated. The proposed model is validated by comparison with an analytical one available for a compressed masonry wall with "standard" viscoelastic mortar joints. Effect induced by microcracks is also highlighted by comparison with uncracked masonry. At last, results provided by the proposed model can be considered to be rigorous solution improving on dilute estimates for the creep behavior of microcracked mortar and demonstrating the interest to not neglect both cracks interactions and creep of bricks units.
\end{abstract}

\section{Keywords}

masonry, creep, microcracks, finite elements, continuum micromechanics, Ponte-Castañeda \& Willis model

\section{Introduction}

The rich heritage of historic masonry buildings in different parts in the world bears the idea that masonry is one of the oldest building materials used by man. Moreover, now days, it is still the most widely used building material. Several models have been developed and presented in the literature for studying and predicting the behavior of masonry structures. Depending on the level of accuracy and simplicity required, either macro- (classical no-tension models [1-8] or micro-modeling [9-13] strategies can be used for this purpose. On the other hand, some authors have combined homogenization techniques with a continuum damage mechanics approach [14-16]. Other authors $[17,18]$, have defined suitably macroscopic yield failure surfaces. Macro-approaches obviously require a preliminary mechanical characterization of the model, based on experimental laboratory or in situ tests $[19,20]$. In studies based on micro-analysis, two main approaches have been used: the simplified approach, which is the more refined, and the detailed micro-modeling approach. Simplified methods consist in modeling the bricks, mortar and interface separately by adopting suitable constitutive laws for each component. This approach gives highly accurate results, especially at local level. Several authors [21-26] have established that the interface elements reflect the main interactions occurring between bricks and mortar. A simplified micro-model is an intermediate approach, where the properties of the mortar and the interface are lumped into a common element, while expanded elements are used to model the brick units. However, this model reduces the computational cost of the analysis, some accuracy is obviously lost. 
Although creep effects in masonry are far from being negligible, in the literature, little attention [27-29] is devoted to the prediction of the macroscopic creep behavior of masonry under sustained loads. In this context, [30-33] proposed models to predict creep coefficients according to the properties of each masonry constituent. These models are based on analytical or numerical homogenization using finite elements method (FEM) in order to deduce the macroscopic creep of undamaged (without cracks) masonry. Moreover, most of these studies, except some works such as Cecchi and Taliercio's paper [31], neglects the brickwork creep which can be argued by the fact that most of the creep effects take place in the bed joints, which are responsible for 60 to $80 \%$ of masonry creep even if their volume fraction represent only $20 \%$ of the brickwork [34]. Nevertheless, the creep deformation of the brick layers may be far from being negligible.

One of the objects of this work is to account for creep of each masonry constituents (brick units and mortar joints). Concerning the creep behavior of traditional mortar, various rheological models namely the USBR, Feng, Ross, typical and modified versions of the Burgers and Modified-Maxwell models may be investigated [35, 36]. Besides, it is well known that cracking is frequently concentrated in mortar joints in case of historical masonry and, in particular, in case of in-plane shear actions with low compression levels. This work takes into account presence of microcracks in the viscoelastic mortar joints. It is interesting to note that in the literature, there exist several approaches accounting for damage in viscoelastic materials $[36,37]$ based for example on a coupling between continuum damage mechanics and viscoelasticity through the generalized Kelvin Voigt model [37] in order to describe the long-term creep of gypsum rock. The main disadvantage of this model is that it requires experimental investigation [36] or computational efforts to resolve nonlinear equation [37] function of internal damage variables. In the works of Nguyen, Dormieux et al. [38, 39], the effective behavior of microcracked linear viscoelastic concrete was derived from a combination of the Griffith's theory [40] and the Eshelby-based homogenization scheme [41]. The undamaged concrete was assumed to obey to the typical Burgers model. In Choi et al. [35], an experimental study was carried in order to investigate the creep of masonry. A number of rheological models (USBR, Feng, Ross, typical Burgers, Modified Maxwell) are examined to assess their ability to predict the creep of masonry. It was proved that the Modified Maxwell model is the most accurate.
According to this result [35], only the Modified Maxwell model (a parallel combination of the Maxwell model and a spring) is adopted in this paper to describe the creep of the mortar joints. Similarly to the mortar and for the sake of simplicity, bricks are assumed to follow the Generalized Maxwell (GM) model. Now, for microcracked mortar, by contrary to recent works Rekik et al. [42-44] and Nguyen et al. $[38,45]$ adopting respectively the dilute and dilute/ Mori-Tanaka schemes in order to estimate the effective creep function of the mortar, it is proposed in this paper to adopt a rigorous homogenization model - the PonteCastañeda \& Willis scheme [46] - accounting for cracks interactions. Indeed, it is worth noting that even the dilute scheme is useful for dilute concentrations of cracks, it has been demonstrated in Dormieux and Kondo [47, 48] that its estimates coincide with those derived from the MoriTanaka (MT) scheme [41]. Moreover, they are close to the Ponte-CastaedaWillis $(\mathrm{PCW})$ estimates for crack density parameter $d_{c} \leq 0.15$ [49]. However the MT predictions violate rigorous bounds of the Hashin-Shtrikmann (HS) [41] type for composites with isotropic distributions of randomly oriented cracks [46]. It is worth noting that the adopted parameter $d_{c}$ allows the measurement of the effect of cracks on the bulk and shear moduli of the considered microcraked material. According to [48], $d_{c}$ is the relevant parameter with regard to the elastic energy as the internal variable governing the effect of damage evolution instead of the crack surface or its length.

In a second step, it is necessary to determine the global behavior of the masonry with viscoelastic bricks and microcracked viscoelastic mortar. This step relies on homogenization technique based on the assumption that a statistically homogeneous medium represented by a 'representative volume element' RVE, or a material with periodic structure represented by a 'repeated unit cell' RUC, can be defined. The RVE, firstly used by Hill [50], corresponds to a microstructural subregion which is representative of the entire sub scale. Generally, the choice or the modeling of the RVE or RUC affects the homogenization results. Indeed the RVE depends on the investigated morphological or physical property, the contrast in the properties of the constituents, and their volume fractions. For given wanted precision and number of realizations, it is possible to provide a minimal volume size for the computation of effective properties. For composites, the RVE is the smallest material volume element which must contain a sufficient number of inclusions, which makes the effective moduli independent of assumed homogeneous 
forces or displacements on the RVE boundary. For regular masonry, as it is the case in the present work, it is useful to consider the pattern which is repeated periodically inside the masonry structure and to apply numerical periodic homogenization approach using FEM. At last, the obtained orthotropic effective properties are used to compute the behavior of a masonry wall subjected to compressive loadings [18, 42, 43].

The layout of the paper is as follows. The first step (see Fig. 1) of the proposed model is detailed in Section 2.

A recall of the $P C W$ estimates for effective moduli of a microcracked media with isotropic distributions of randomly oriented (closed or opened) cracks is provided. After that the identification procedure of the $M M$ 's six parameters for the microcracked mortar at short and long terms allowing the expression of its creep function is presented. The last part in Section 2 shows an illustrative example for the followed methodology in step 1. Section 3 deals with the second step (Fig. 1) of the proposed model based on numerical periodic homogenization of regular masonry accounting for creep of both brick units and mortar joints (bricks are assumed to be safe unlike mortar). Section 4 validates the proposed numerical model by comparison with analytical expression for local displacement at the top of a compressed masonry wall with rigid bricks and 'standard' viscoelastic mortar joints. At last, the relevance of the proposed model is illustrated by investigating the case of a masonry wall subjected to compressive loadings.

\section{Instantaneous global properties of viscoelastic microcracked mortar (step 1)}

The results of brittle fracture mechanics - the Griffith's theory - could be useful if we move from the real temporal space to the symbolic one due the Laplace-Carson (LC) transform. In the symbolic space, the apparent behavior of the mortar is linear elastic. This procedure allows the use of expressions available in the literature for the displacement's jump induced by the crack [43]. Assuming again that the displacement jump field depends linearly on the macroscopic stress, it is possible to define an effective linear behavior for the microcracked mortar in the symbolic space using one of the available homogenization schemes (dilute, Mori-Tanaka, self-consistent, $P C W$ or others [41]). To determine the global behavior in the real space time, it is possible to apply the inverse of the LC transform in some simple cases. It is then interesting to approach in the symbolic space, at least in short and long terms, the symbolic effective stiffness (or compliance) by an existing rheological model. For example, if the undamaged mortar behaves as the Modified Maxwell model, we try to approach the symbolic effective behavior of the corresponding microcracked mortar by the same model. After validation of this approximation at short and long terms, the inversion of the apparent effective stiffness will be straightforward. Therefore, the effective behavior of the micro-cracked viscoelastic mortar could be expressed in the real space time.
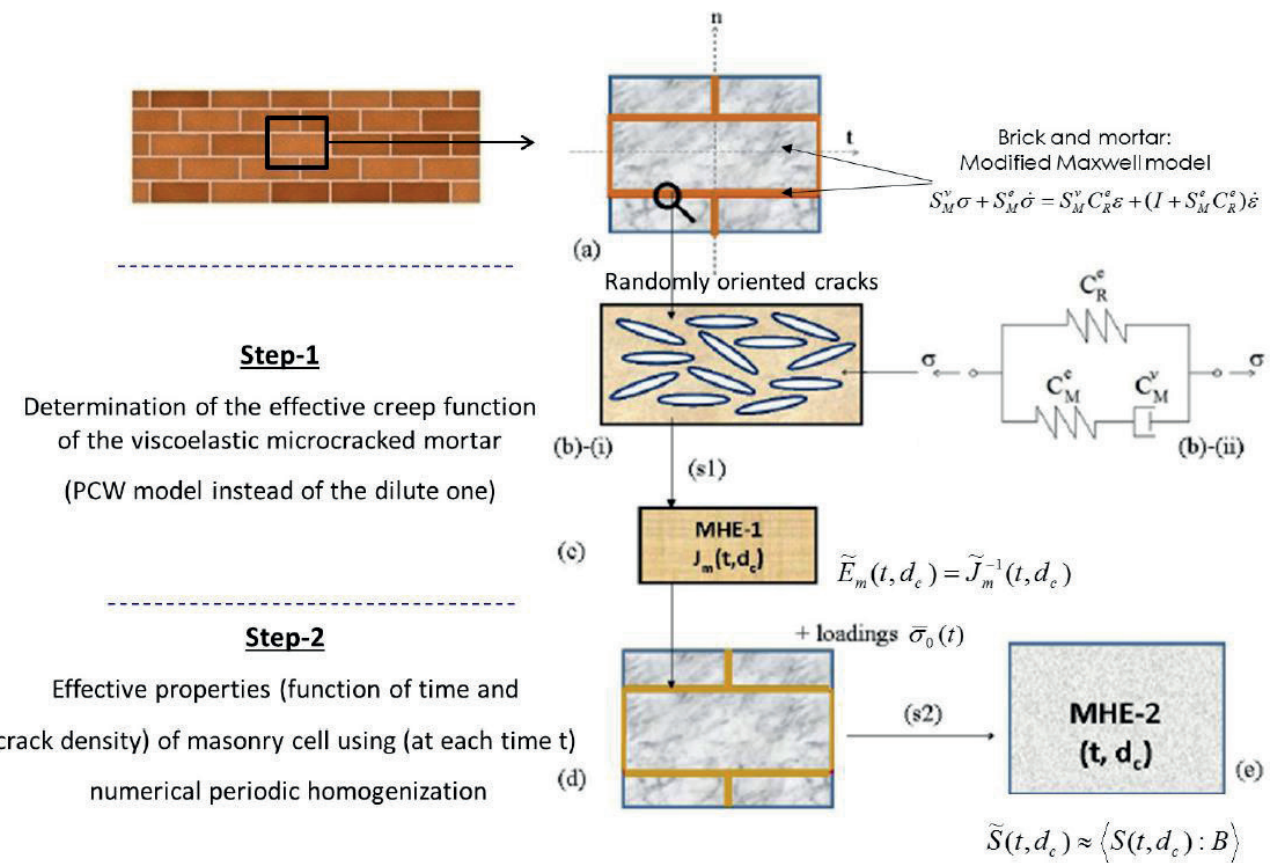

Fig. 1 Basic steps followed by the propose model: (s1) and (s2) 
In this work, in the symbolic space, the Ponte-Castañeda and Willis homogenization scheme $(P C W)[46]$ is adopted for its ability of properly accounting for interactions and influence of spatial distribution of microcracks by contrary to the dilute scheme.

\subsection{Ponte-Castañeda \& Willis model}

We distinguish the case of closed from that of opened cracks as shown in the following

\subsubsection{Closed cracks}

We assume that the mortar contains a set of closed cracks with unit normal $n$ and that the contact between the two crack lips is frictionless. In contrast to open cracks, it is necessary to take into account the fact that a compressive normal stress can be transmitted through the contact of the crack lips, while the shear stress in the crack plane remains zero. Adopting a continuum micromechanics approach, the closed crack is represented by a flat ellipsoidal inhomogeneity filled with an isotropic fictitious material having a zero shear modulus, $\mu_{c}=0$ and a nonzero bulk modulus, $k^{c} \neq 0$. Accordingly $C^{c}=3 k^{c} J$ where $J$ is the spherical fourth-order projector. For randomly oriented closed cracks requiring an averaging process on crack orientations, the effective symbolic bulk and shear moduli read

$$
\left\{\begin{array}{l}
\tilde{k}_{P C W}^{*}=\tilde{k}_{m}^{*} \\
\tilde{\mu}_{P C W}^{*}=\tilde{\mu}_{m}^{*}\left(1-\frac{480 d_{c}\left(1-v_{m}^{*}\right)}{225\left(2-v_{m}^{*}\right)+64 d_{c}\left(4-5 v_{m}^{*}\right)}\right),
\end{array}\right.
$$

where $v_{m}^{*}$ is the symbolic Poisson's ratio, $k_{m}^{*}$ and $\mu_{m}^{*}$ are respectively the matrix's symbolic bulk and shear moduli.

\subsubsection{Open cracks}

When all microcracks in the RVE (Representative Volume Element) are open, the global symbolic deformation is elastic and the effective symbolic stiffness tensor $\tilde{C}$ read

$$
\tilde{C}=3 \tilde{k}_{P C W}^{*} J+2 \tilde{\mu}_{P C W}^{*} K,
$$

where the effective bulk and shear moduli are given in the symbolic space by $K$ is the deviatoric fourth-order projector defined by $K=I-J$ and symbolic properties of the safe (uncracked) matrix are given by

$$
\begin{aligned}
& \left\{\begin{array}{c}
\tilde{k}_{P C W}^{*}=\tilde{k}_{m}^{*}\left(1-\frac{48 d_{c}\left(1-\left(v_{m}^{*}\right)^{2}\right)}{27\left(1-2 v_{m}^{*}\right)+16 d_{c}\left(1+v_{m}^{*}\right)^{2}}\right) \\
\tilde{\mu}_{P C W}^{*}=\tilde{\mu}_{m}^{*}\left(1-\frac{480 d_{c}\left(1-v_{m}^{*}\right)\left(5-v_{m}^{*}\right)}{675\left(2-v_{m}^{*}\right)+64 d_{c}\left(4-5 v_{m}^{*}\right)\left(5-v_{m}^{*}\right)}\right) \\
\mu_{m}^{*}(p)=\mu_{R}^{e}+\frac{1}{\frac{1}{\mu_{M}^{e}}+\frac{2}{p \eta_{m}^{d}}}, k_{m}^{*}(p)=k_{R}^{s e}+\frac{1}{\frac{1}{k_{M}^{e}}+\frac{3}{p \eta_{m}^{e}}}
\end{array}\right. \\
& v_{m}^{*}=\frac{3 k_{m}^{*}-2 \mu_{m}^{*}}{6 k_{m}^{*}+2 \mu_{m}^{*}} .
\end{aligned}
$$

\subsubsection{Opening-closure transition criteria}

A crack is opened means that its lips are free of stress which can be expressed as follows

$$
\sigma_{x y}=\sigma_{y y}=0: \quad\left[u_{y}\right] \geq 0 \text {, }
$$

where $\left[u_{y}\right]$ is the displacement jump across the upper and lower crack surfaces. The last condition implies that the asymptotic stress at infinity is a tensile stress $\sigma_{y y}^{\infty} \geq 0$ otherwise crack closure occurs. Accordingly, a frictionless closed crack, which is a model of unilateral contact, can be defined as follows

$\sigma_{x y}=0, \sigma_{y y} \leq 0: \quad\left[u_{y}\right]=0$.

The transition between the two regimes is defined by the relations

$\sigma_{x y}=\sigma_{y y}=0: \quad\left[u_{y}\right]=0$.

\subsection{Identification procedure of existent rheological model at short and long times}

Whatever the state of the crack (open or closed), the following described identification procedure remains available. Only mortar properties at the macroscopic scale change owing to the state of the crack and the adopted homogenization scheme accounting for the presence of cracks.

The series expansion of the $P C W$ estimates for the mortar's bulk's and shear symbolic modulus at the vicinity of $p=0$ gives

$$
\begin{aligned}
& \tilde{k}_{P C W}^{*}=\tilde{k}_{0}^{0}+\tilde{k}_{0}^{1} p+O\left(p^{2}\right) \\
& \tilde{\mu}_{P C W}^{*}=\tilde{\mu}_{0}^{0}+\tilde{\mu}_{0}^{1} p+O\left(p^{2}\right)
\end{aligned}
$$

and at the vicinity of $p=\infty$, it reads 
$\tilde{k}_{P C W}^{*}=\tilde{k}_{\infty}^{0}+\frac{\tilde{k}_{\infty}^{1}}{p}+O\left(1 / p^{2}\right)$

$\tilde{\mu}_{P C W}^{*}=\tilde{\mu}_{\infty}^{0}+\frac{\tilde{\mu}_{\infty}^{1}}{p}+O\left(1 / p^{2}\right)$.

Hereafter, parameters $\tilde{k}_{0}^{0}, \tilde{k}_{0}^{1}, \tilde{k}_{\infty}^{0}, \tilde{k}_{\infty}^{1}, \tilde{\mu}_{0}^{0}, \tilde{\mu}_{0}^{1}, \tilde{\mu}_{\infty}^{0}, \tilde{\mu}_{\infty}^{1}$ are assumed to be known. For example, for open cracks, using theorems on the initial and final values given by

$\lim _{p \rightarrow 0} f^{*}(p)=\lim _{p \rightarrow \infty} f(t), \lim _{p \rightarrow \infty} f^{*}(p)=\lim _{t \rightarrow 0} f(t)$,

the $P C W$ 's symbolic bulk's and shear's moduli (Eq. (3)) of a non-aging linear viscoelastic (n.a.l.v.) microcracked mortar following the $M M$ 's model can be approached, at least at short and long terms, by expressions (14) provided in [43] available for a mortar with a matrix (uncracked mortar) following the $M M$ 's rheological model

$$
\left\{\begin{array}{l}
k_{M M}^{*}=k_{R}\left(d_{c}\right)+\frac{p k_{M}\left(d_{c}\right) \eta_{M}^{s}\left(d_{c}\right) / 3}{k_{M}\left(d_{c}\right)+p \eta_{M}^{s}\left(d_{c}\right) / 3} \\
\mu_{M M}^{*}=\mu_{R}\left(d_{c}\right)+\frac{p \mu_{M}\left(d_{c}\right) \eta_{M}^{d}\left(d_{c}\right) / 2}{\mu_{M}\left(d_{c}\right)+p \eta_{M}^{d}\left(d_{c}\right) / 2}
\end{array} .\right.
$$

The series expansion of Eq. (10) at the vicinity of $p=0$ gives $k_{M M}^{*}=k_{R}^{s e}+\frac{\eta_{m}^{s}}{3} p+O\left(p^{2}\right)$,

$\mu_{M M}^{*}=\mu_{k}^{e}+\frac{\eta_{m}^{d}}{2} p+O\left(p^{2}\right)$

At the vicinity of $p=\infty, M M$ 's symbolic moduli can be approximated by

$$
\begin{aligned}
& k_{M M}^{*}=\left(k_{R}^{s e}+k_{m}^{s e}\right)-3 \frac{\left(k_{m}^{e}\right)^{2}}{\eta_{M}^{s} p}+O\left(1 / p^{2}\right), \\
& \mu_{M M}^{*}=\left(\mu_{k}^{e}+\mu_{M}^{e}\right)-2 \frac{\left(\mu_{M}^{e}\right)^{2}}{\eta_{M}^{d} p}+O\left(1 / p^{2}\right) .
\end{aligned}
$$

The Eq. (7) = Eq. (11), Eq. (8) = Eq. (12) allow the identification of the following expressions for the six effective $M M$ 's parameters for the $\mathrm{HEM}_{m}$ (the homogeneous equivalent material to the microcracked mortar)

$$
\begin{aligned}
& k_{R}^{s e}=\tilde{k}_{0}^{0}, k_{m}^{s e}=\tilde{k}_{\infty}^{0}-k_{R}^{s e}, \eta_{M}^{s}=3 \tilde{k}_{0}^{1}, \\
& \mu_{k}^{e}=\tilde{\mu}_{0}^{0}, \mu_{M}^{e}=\tilde{\mu}_{\infty}^{0}-\mu_{k}^{e}, \eta_{M}^{d}=2 \tilde{\mu}_{0}^{1},
\end{aligned}
$$

respectively for spherical and deviatoric parts.
The characteristic times of the spherical and deviatoric parts of the Modified Maxwell model followed by the microcracked viscoelasticortar are respectively

$\tau_{M M}^{s}\left(d_{c}\right)=\frac{\eta_{M}^{s}\left(d_{c}\right)\left(k_{R}\left(d_{c}\right)+k_{M}\left(d_{c}\right)\right)}{3 k_{R}\left(d_{c}\right) k_{M}\left(d_{c}\right)}$

$\tau_{M M}^{d}\left(d_{c}\right)=\frac{\eta_{M}^{d}\left(d_{c}\right)\left(\mu_{R}\left(d_{c}\right)+\mu_{M}\left(d_{c}\right)\right)}{2 \mu_{R}\left(d_{c}\right) \mu_{M}\left(d_{c}\right)}$.

At last, the approximate creep function of a microcracked mortar which matrix follows the $M M$ 's model reads in Eq. (15).

$$
\begin{aligned}
& \mathrm{J}_{M M}^{a p p}\left(t, d_{c}\right)=\frac{1}{9 k_{R}\left(d_{c}\right)}\left(1-\frac{k_{M}\left(d_{c}\right)}{\left(k_{R}\left(d_{c}\right)+k_{M}\left(d_{c}\right)\right)} e^{-t / \tau_{M M}^{s}\left(d_{c}\right)}\right) \\
& +\frac{1}{3 \mu_{R}\left(d_{c}\right)}\left(1-\frac{\mu_{M}\left(d_{c}\right)}{\left(\mu_{R}\left(d_{c}\right)+\mu_{M}\left(d_{c}\right)\right)} e^{-t / \tau_{M M}^{d}\left(d_{c}\right)}\right) .
\end{aligned}
$$

\subsection{Illustrative example}

In order to illustrate the above described identification procedure whether for mortar with open or closed cracks, it is proposed to consider hereafter the case of a microcracked mortar which properties at the safe state are given in Table 1.

\subsubsection{Closed cracks}

Dilute and $P C W$ estimates for effective elastic and viscous properties (explicit functions of the parameter $d_{c}$ ) of the considered microcracked viscoelastic mortar with closed cracks are provided in the Table 1. For closed cracks, we recall that the bulk's modulus of the microcracked mortar coincides with that of the safe matrix which is independent from the crack density. Accordingly, the $P C W$ and dilute estimates for the spherical part of the mortar's behavior coincide and are also independent from crack density $d_{c}$.

As shown on Table 2, only expressions of the effective properties related to the deviatoric part of the mortar's behavior differ with the adopted predictive model. Accordingly, also the $P C W$ estimate for the mortar's "effective Poisson's ratio" is a function of the crack density (but it is time free).

Table 1 Elastic and viscous moduli of the mortar (one term, $n=1$ ) tested by Brooks et al. [30, 31]

\begin{tabular}{ccccc}
\hline & $E_{0}(\mathrm{MPa})$ & $N$ & $e_{i}$ & $\tau_{M}$ (days) \\
\hline Mortar & 7700 & 0.2 & 0.7602 & 7.1 \\
\hline
\end{tabular}


Table 2 Closed cracks: identified MM's parameters for the microcracked mortar at the macroscopic scale (effective properties) using the PCW and dilute homogenization models

\begin{tabular}{lccc}
\hline & $k_{R}(\mathrm{MPa})$ & $k_{M}(\mathrm{MPa})$ & $\eta_{s}(\mathrm{MPa} . \mathrm{s})$ \\
PCW & 1025.81 & 3251.97 & $5.984610^{9}$ \\
model & $\mu_{R}(\mathrm{MPa})$ & $\mu_{M}(\mathrm{MPa})$ & $\eta_{d}(\mathrm{MPa} . \mathrm{s})$ \\
& $-769.358+\frac{3245.73}{2.10938+d_{c}}$ & $-2438.98+\frac{10289.4}{2.10937+d_{c}}$ & $-2.9923310^{9}+\frac{1.2623910^{10}}{2.10938+d_{c}}$ \\
\hline $\begin{array}{l}\mu_{R} \\
\text { Model }\end{array}$ & $\frac{\mu_{M}}{269.358}$ & $\frac{2438.97}{1+0.948148 d_{c}}$ & $\frac{\eta_{d}}{1+0.94823310^{9}}$ \\
\hline
\end{tabular}

This expression is obtained by an exact inversion of the Laplace-Carson transform as follows

$v_{P C W}^{*}\left(p, d_{c}\right)=\frac{3 \tilde{k}_{P C W}^{*}-2 \tilde{\mu}_{P C W}^{*}}{6 \tilde{k}_{P C W}^{*}+2 \tilde{\mu}_{P C W}^{*}}$

accordingly $v_{P C W}\left(d_{c}\right)=1-\frac{2.8125}{3.51562+d_{c}}$,

which is not heavy to carry out by contrary to the LC inversion [51] of $P C W$ estimate for the mortar's effective creep function.

Fig. 2(a) demonstrates that the mortar's "effective Poisson's ratio" increases with the increase of the crack density in the case of closed cracks. It remains lower than 0.5 showing that the mortar is far from to be incompressible. In case of closed cracks, the damage is assumed to do not evolve [47], accordingly, hereafter the crack density dc is assumed to be constant.

Fig. 3 reports time-evolution of the mortar's Young's modulus for different crack densities values $\left(d_{c}=0,0.2\right.$, $0.55,1)$. Qualitatively, it shows that the PCW estimate decreases rapidly with time $t$ during a period of about $5.10^{6} \approx 58$ days. In a second stage, it stabilizes or reaches a non-null asymptotic limit. This trend is also available for $d_{c}=1$. Quantitatively, this asymptotic limit decreases with the increase of the crack density.

On Fig. 4 are depicted the $P C W$ and dilute estimates for the mortar's Young's moduli evolutions with crack densities at times $t=20$ and 1000 days. It can be observed that $P C W$ and dilute models provide close estimates for the effective modulus $\tilde{E}_{P C W}\left(t, d_{c}\right)$ for a crack density $0 \leq d_{c} \leq 0.25$. Beyond this interval, the percent error

$E_{r r}(\%)=100\left(\frac{\tilde{E}_{P C W}-\tilde{E}_{D I L}}{\left(\tilde{E}_{P C W}+\tilde{E}_{D I L}\right) / 2}\right)$.

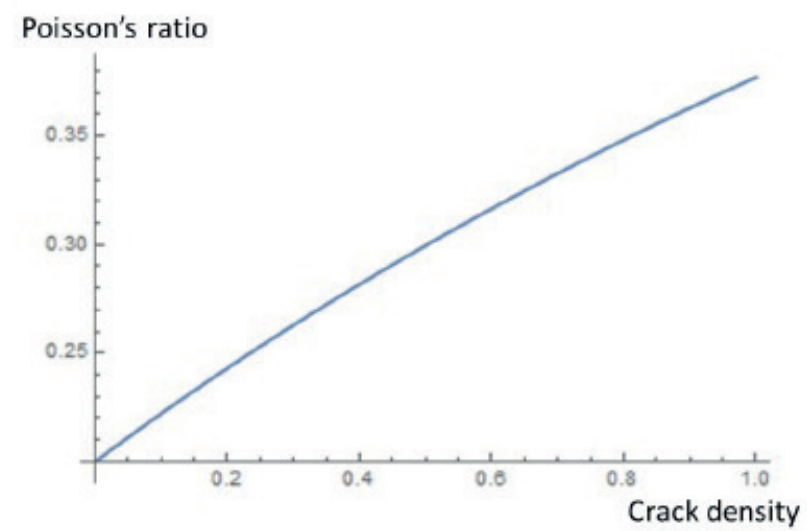

(a)

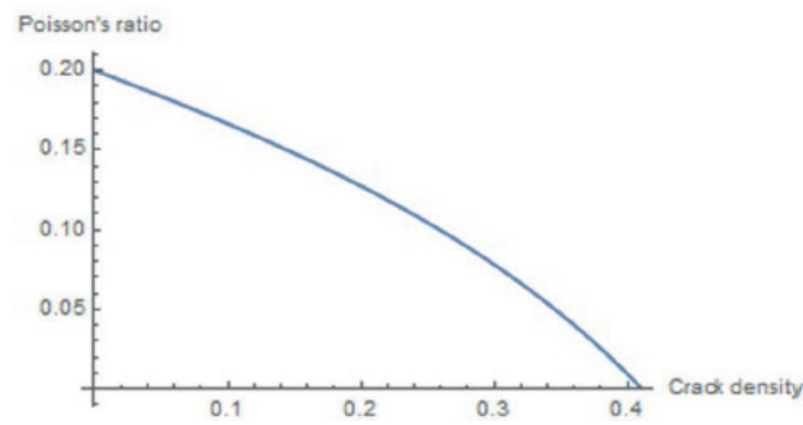

(b)

Fig. 2 Evolution of the PCW estimate for the effective "Poisson's ratio" of the microcracked mortar with crack density dc in the case of closed (a) and open (b) cracks

Between these estimates increases to exceed $10 \%$ for $d_{c} \geq 0.55$ as shown on Fig. 5 . It can also be remarked that the $P C W$ effective estimates are softer than the dilute ones which is an expected result due to the fact that the $P C W$ model accounts for cracks interactions by contrary to the dilute scheme. For mortar with closed cracks, it can be concluded that dilute estimate remains acceptable for every time $t$ and crack density $d_{c}<0.55$ (see Figs. 5 and 6). Otherwise this model highly overestimates the effective Young's modulus of microcracked mortar. Besides, 


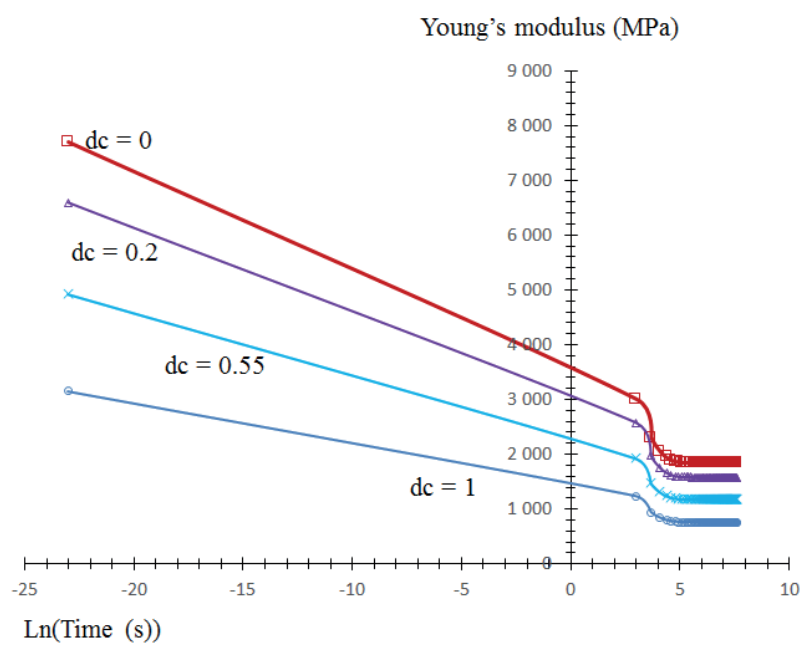

Fig. 3 Closed cracks: PCW estimates for the evolution of the mortar's effective Young's modulus versus "Ln(time)"

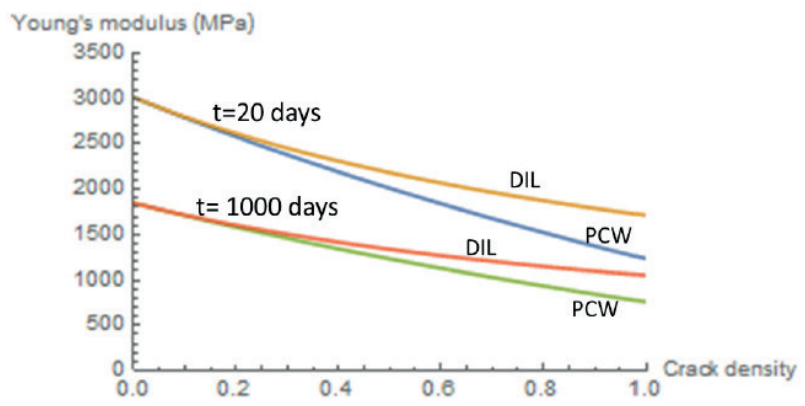

Fig. 4 Closed cracks: comparison between PCW and dilute estimates for the microcracked mortar's effective Young's modulus ( $t=1000$ days) versus crack density

cumulative errors for both viscoelastic constituents (mortar and bricks) if bricks are also assumed to be microcracked could lead to (highly) stiffer macroscopic estimates for the masonry.

\subsubsection{Open cracks and time-dependent crack density}

By contrary to the case of closed microcracks, both spherical and deviatoric parts of the mortar's behavior are affected by the presence of open cracks. Accordingly, the six effective mortar's (elastic and viscous) parameters identified after averaging process on randomly oriented cracks based on the $P C W$ model are different from those provided by the dilute scheme. Table 3 provides these parameters for both $P C W$ and dilute models when the microcracked mortar's properties at the safe (uncracked) state are given in Table 1.

Based on these identified parameters, it is possible to calculate explicitly the effective creep function and hence the mortar's Young's modulus and its effective "Poisson's ratio" in the case of open cracks.

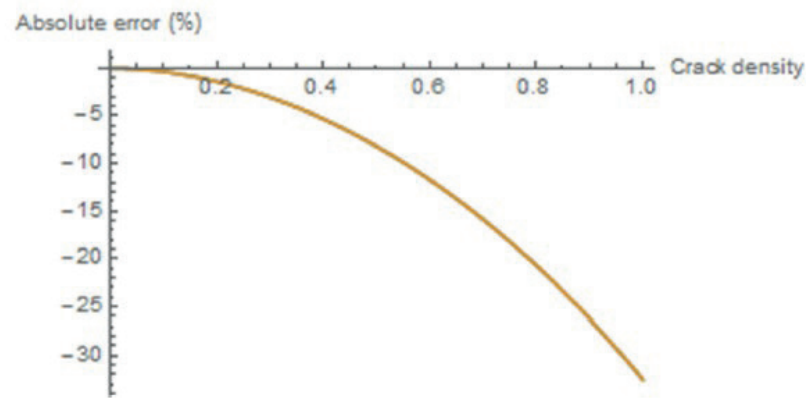

Fig. 5 Closed cracks: percent error between PCW and dilute estimates for the microcracked mortar's effective Young's modulus $(t=20$ and 1000 days) versus crack density

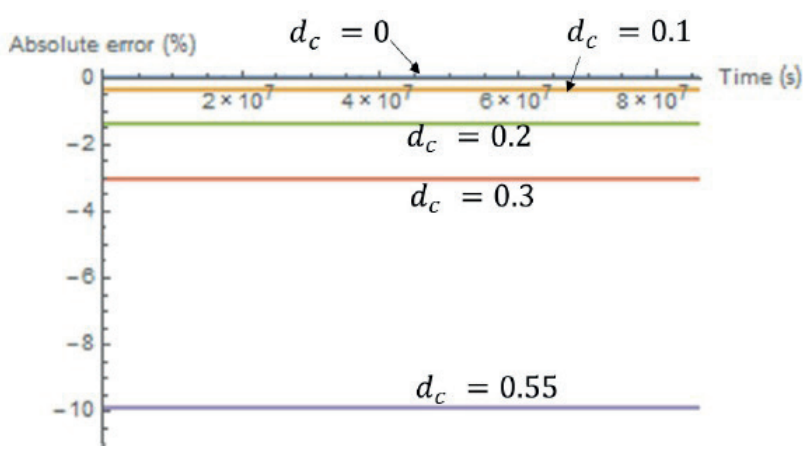

Fig. 6 Closed cracks: percent error between PCW and dilute estimates for the microcracked mortar's effective Young's modulus versus time

$\tilde{v}_{P C W}=\frac{-0.185394+\left(0.369141+0.2 d_{c}\right) d_{c}}{-0.926971+d_{c}\left(0.369141+d_{c}\right)}$

By contrary to the case of closed cracks, Fig. 2(b) shows that the mortar's effective "Poisson's ratio" decreases with the increase of density $d_{c}$. The later should not exceed 0.41 otherwise the mortar's effective "Poisson's ratio" tends towards negative values which is aberrant. In the following, for the case of microcracked mortar with open cracks, we assume that the condition $d_{c} \leq 0.41$ is fulfilled. Moreover, for the sake of showing the trends, we assume firstly that crack density is constant. Secondly, since damage evolves in mortar (or more generally a media) with open cracks, than crack density is assumed to evolve following a power-law time function as prescribed by [54] for the sake of simplicity and as a first assumption.

Constant crack density. Fig. 7 shows that the $P C W$ estimates for the mortar's Young's modulus decreases with the increase of damage but does not vanish for $d_{c} \leq 0.4$. Moreover as predicted by the dilute scheme [42], the $P C W$ estimates stabilizes beyond $t \geq 2.107 \approx 231$ days and tend towards none null asymptotic limits even if the $P C W$ model provides softer estimates than the dilute ones for $t \leq 1000$ days (see Fig. 8). The huge difference between the $P C W$ and dilute estimates mainly beyond $d_{c}=0.2$ (see Figs. 9 and 10) 
Table 3 Open cracks: identified $M M$ 's parameters for the microcracked mortar at the macroscopic scale using the PCW and dilute homogenization models

\begin{tabular}{|c|c|c|c|}
\hline \multirow{3}{*}{$\begin{array}{l}\text { PCW } \\
\text { model }\end{array}$} & $k_{R}(\mathrm{MPa})$ & $k_{M}(\mathrm{MPa})$ & $\eta_{s}(\mathrm{MPa} . \mathrm{s})$ \\
\hline & $\begin{array}{c}-1025.81+\frac{1442.55}{0.703125+d_{c}} \\
\mu_{R}(\mathrm{MPa})\end{array}$ & $\begin{array}{c}-3251.97+\frac{4573.08}{0.703125+d_{c}} \\
\mu_{M}(\mathrm{MPa})\end{array}$ & $\begin{array}{c}-5.984610^{9}+\frac{8.4159310^{9}}{0.703125+d_{c}} \\
\eta_{d} \text { (MPa.s) }\end{array}$ \\
\hline & $-769.358+\frac{2028.58}{1.31836+d_{c}}$ & $-2438.98+\frac{6430.89}{1.31836+d_{c}}$ & $-2.9923310^{9}+\frac{7.8899310^{9}}{1.31836+d_{c}}$ \\
\hline \multirow{6}{*}{$\begin{array}{l}\text { Dilute } \\
\text { model }\end{array}$} & $k_{R}$ & $k_{M}$ & $\eta_{s}$ \\
\hline & 1025.81 & 3251.97 & $5.9846610^{9}$ \\
\hline & $\overline{1+2.84444 d_{c}}$ & $\overline{1+2.84444 d_{c}}$ & $\overline{1+2.84444 d_{c}}$ \\
\hline & $\mu_{R}$ & $\mu_{M}$ & $\eta_{d}$ \\
\hline & 769.358 & 2438.97 & $2.9923310^{9}$ \\
\hline & $\overline{1+1.51704 d_{c}}$ & $\overline{1+1.51704 d_{c}}$ & $\overline{1+1.51704 d_{c}}$ \\
\hline
\end{tabular}

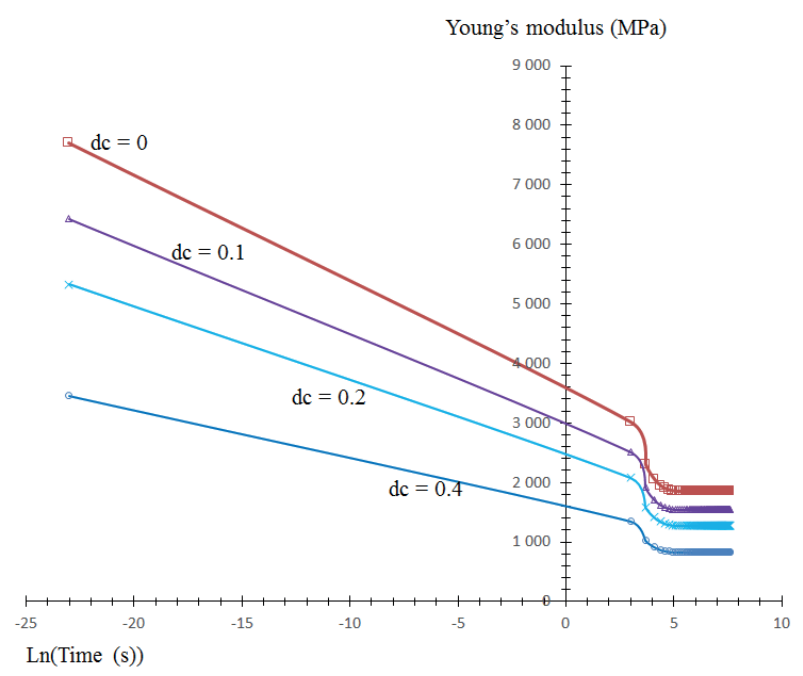

Fig. 7 Open cracks: PCW estimates for the mortar's Young's modulus versus "Ln(Time(s))" for different crack densities

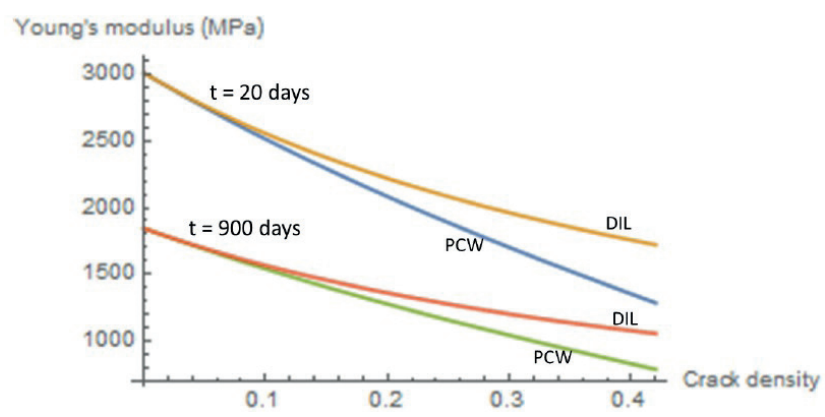

Fig. 8 Open cracks: PCW and dilute estimates for the microcracked mortar's Young's modulus versus crack density at instants $t=20$ and 1000 days

demonstrates that the presence of open cracks implies a high interaction level between the micro-cracks by contrary to the same density of closed cracks. Accordingly, for damaged mortar with open cracks it is important and more rigorous to assess its effective Young's modulus by means of the $P C W$ model instead of the dilute one if $d_{c}>0.2$.

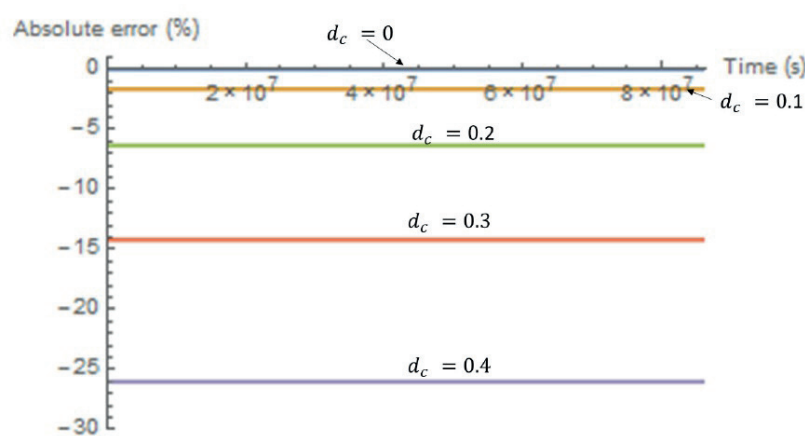

Fig. 9 Open cracks: percent errors between PCW and dilute estimates for the micro-cracked mortar's Young's modulus versus crack density either for $t=20$ and 1000 days

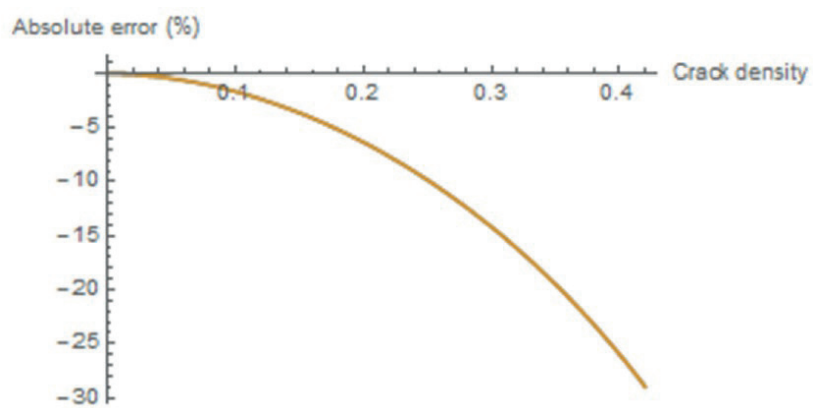

Fig. 10 Open cracks: percent errors between PCW and dilute estimates for the microcracked mortar's Young's modulus with different crack densities $\left(d_{c}=0,0.1,0.2,0.3\right.$ and 0.4$)$ versus time

Time-dependent crack density evolution. As noted above, for the sake of simplicity, the crack density is assumed to accumulate in the following form as proposed by Shrive et al. [52]

$$
d_{c}(t)=\sum_{i=t_{0}}^{t} \frac{100 \eta}{\tau_{D}}\left(\frac{t}{\tau_{D}}\right)^{n},
$$

where $\tau_{D}$ is a constant damage time which refers to the time where most damage would occur. The coefficients are taken here as $\tau_{D}=800, \eta=0.3$, and $n=10 . d_{c}(t)$ represents 
the level of damage accumulated from the time at which damage starts, $t_{0}$, to the time of evaluation $t$. In the calculations here, damage is assumed to begin at 400 days. The rate of damage accumulation with this model is slow initially, but accelerates over time, as shown on Fig. 11 reporting Fig. 4.4 in [52].

For the sake of showing the trends and effects, quite considerable damage is assumed to occur in a relatively short time in this example. Here damage factor attains about 0.33 after 1000 days with the damage starting at 400 days. Damage initially accumulates at a very low rate but then increases rapidly [52].

Fig. 12 shows a rapid decrease of the mortar's Young's modulus for $t \leq 5.10^{7}$ (s) $\approx 578$ (days). After that it tends towards an asymptotic limit of about 2000 (MPa). At a third step, it decreases rapidly for $t \geq 8.10^{7}$ (s). It can also be observed that for the considered time power-law crack density, dilute and $P C W$ estimates for time evolution of the mortar's effective Young's modulus are close for $t \leq t_{1}=8 \cdot 10^{7}(\mathrm{~s}) \approx 925$ (days) (Fig. 13). This can be argued by the fact that $\mathrm{dc}$ is negligible below $t_{1}$. After that

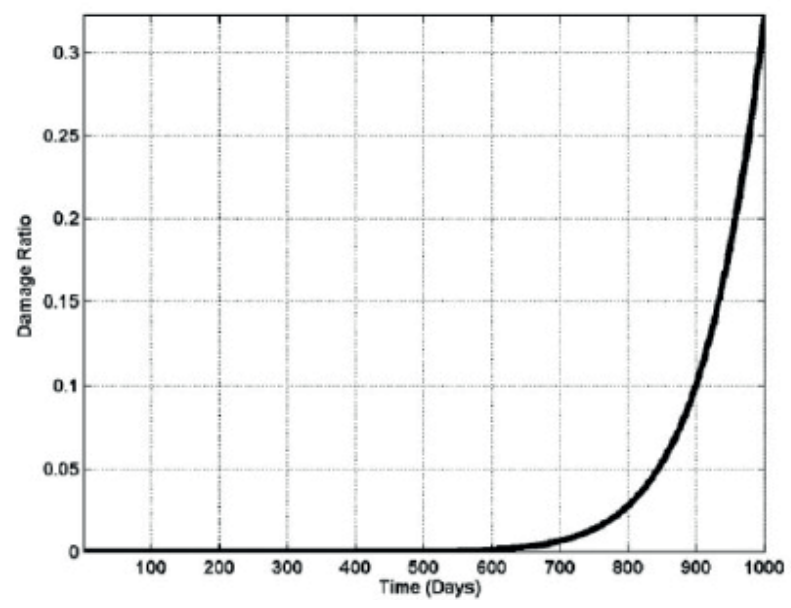

Fig. 11 Non-linear evolution of damage ratio with time [52]

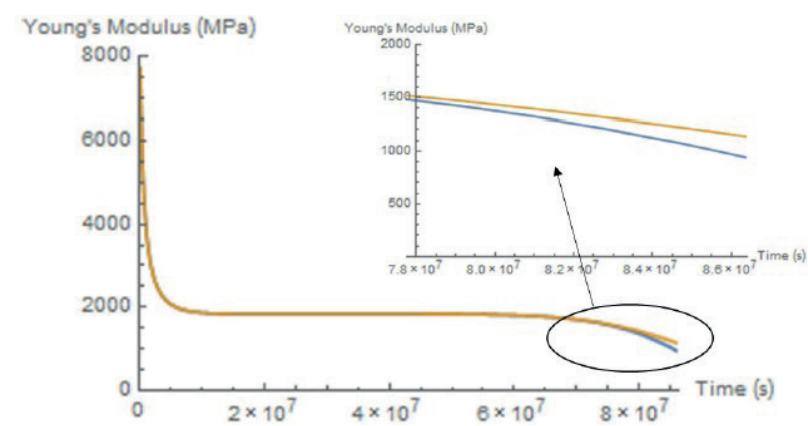

Fig. 12 Comparison between dilute and PCW estimates: time evolutions of the microcracked mortar's Young's modulus with open cracks which crack density is assumed to be a time power-law function

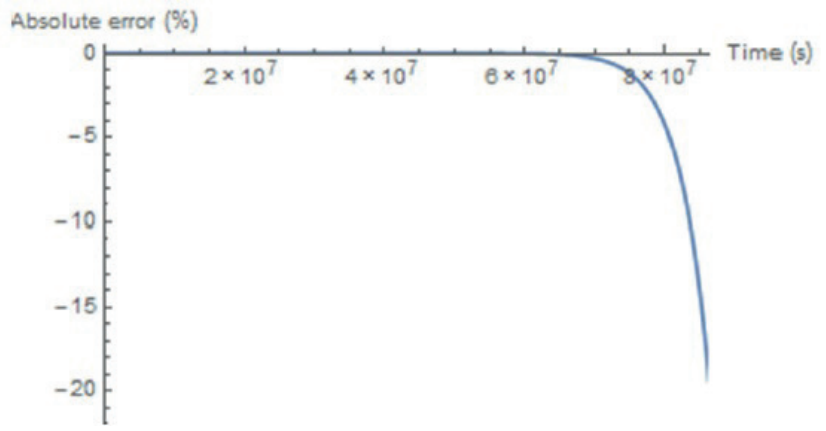

Fig. 13 Open cracks which crack density evolves following a time power-law function: percent error between PCW and dilute estimates

(that means when $t>t_{1}$ ) this damage parameter increases rapidly. Accordingly the mortar's effective Young's modulus decreases rapidly and it can be observed that the $P C W$ estimates are (highly) softer than the dilute ones. Moreover, errors between these estimates exceed $10 \%$ beyond $t_{2}=8.410^{7}(\mathrm{~s}) \approx 972$ (days). This trend is consistent with that observed on Fig. 9

\section{Actual global properties of microcracked masonry using periodic homogenization (step 2): viscoelastic bricks and mortar}

In this part, both of brick and mortar are assumed to follow the Generalized Maxwell (GM) model. For the sake of simplicity, only one term is considered for the GM model [34]. Accordingly rheological model followed by bricks and mortar coincide with the $M M$ 's model which tends towards a "purely" elastic behavior both at the vicinity of 0 or at infinity as shown below

$$
\begin{aligned}
& \left(J_{M M}(t \rightarrow 0)=\frac{1}{9\left(k_{R}+k_{M}\right)}+\frac{1}{3\left(\mu_{R}+\mu_{M}\right)}\right) \\
& \left(J_{M M}(t \rightarrow \infty)=\frac{1}{9 k_{R}}+\frac{1}{3 \mu_{R}}\right) .
\end{aligned}
$$

The mortar's behavior can then be considered elastic and damaged at short and long terms as it evolves with crack density variation. Its behavior, at each time $t$, can be described by two effective parameters: the Poisson's ratio (Eq. (16)) if cracks are closed (respectively, Eq. (17), if cracks are opened) and "Young's modulus" $\tilde{E}_{P C W}\left(t, d_{c}\right)=$ $1 / / \tilde{J}_{P C W}\left(t, d_{c}\right)$ if damaged or $\tilde{E}_{P C W}(t)=1 / \tilde{J}_{P C W}(t)$ otherwise $\left(d_{c}=0\right)$. The considered bricks are assumed to be isotropic, safe (uncracked) with a viscoelastic behavior following the $M M^{\prime}$ 's model with parameters data given in Table 4. 
Table 4 Elastic and viscous moduli of brick (one term, $n=1)[30,31]$

\begin{tabular}{ccccc}
\hline & $E_{0}(\mathrm{MPa})$ & $N$ & $e_{i}$ & $\tau_{M}$ (days) \\
\hline Brick & 17100 & 0.15 & 0.5327 & 33.8 \\
\hline
\end{tabular}

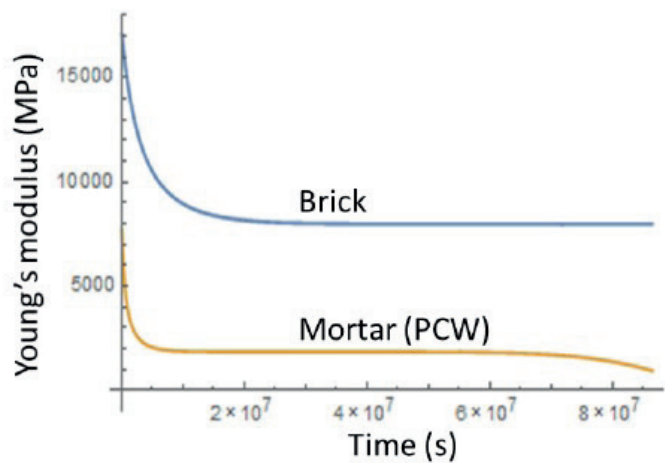

Fig. 14 Time evolutions of the "effective Young's moduli" of the considered viscous brick and mortar

Bricks can than be considered to be "elastic" with a time-dependent "Young's modulus" (see Fig. 14).

$E_{b}(t)=\frac{1}{J_{M M}^{b}(t)}=\frac{7990.83}{1-0.5327 e^{-1.60017 .10^{-7} t}}$

Dimensions of the bricks are the following: height $a=55 \mathrm{~mm}$, width $b=120 \mathrm{~mm}$ and thickness $s=250 \mathrm{~mm}$. Mortar joints are $10 \mathrm{~mm}$ thick. For the viscous rheological model, since the instantaneous Young's moduli $E_{0}$ for the $M M$ 's model is given by $E_{0}=E(t=0)=E_{R}+E_{M}$ where the relaxation moduli is set equal to $E_{M}=e_{i} E_{0}$ then the spring's Young's moduli $E_{R}$ reads $E_{R}=\left(1-e_{i}\right) E_{0}$. ei is a dimensionless parameter.

Now, according to the fact that the considered masonry shows regular arrangement of bricks and mortar's joints, it is possible to consider only a periodic cell and carry out numerical periodic homogenization using FE method. Moreover, as this periodic cell presents two axes of symmetry, normal and tangential directions along the unit vectors $n$ and $t$, respectively, only its quarter (see Fig. 15) will be retained for computation.

When applying a constant macroscopic stress and assuming that the per phase localization tensor $A^{r}$ is time independent as previously mentioned in [43] following the hypothesis of Deudé et al. [53] then the average strain $\varepsilon^{-r}$ per phase $r$ and the masonry's overall behavior reduce respectively to $\bar{\varepsilon}^{r}=A^{r}: \bar{\varepsilon}$ and $\bar{\sigma}=\tilde{C}: \bar{\varepsilon}$ where the overall stiffness is given by $\tilde{C}=\langle C: A\rangle$. Recall that the average strain localization over the periodic cell reads $\langle A\rangle=I$. It is then important to determine components of the localization strain tensor $A_{i j k l}^{r}$. To assess the effective "elastic engineering constants", it is proposed to subject the unit
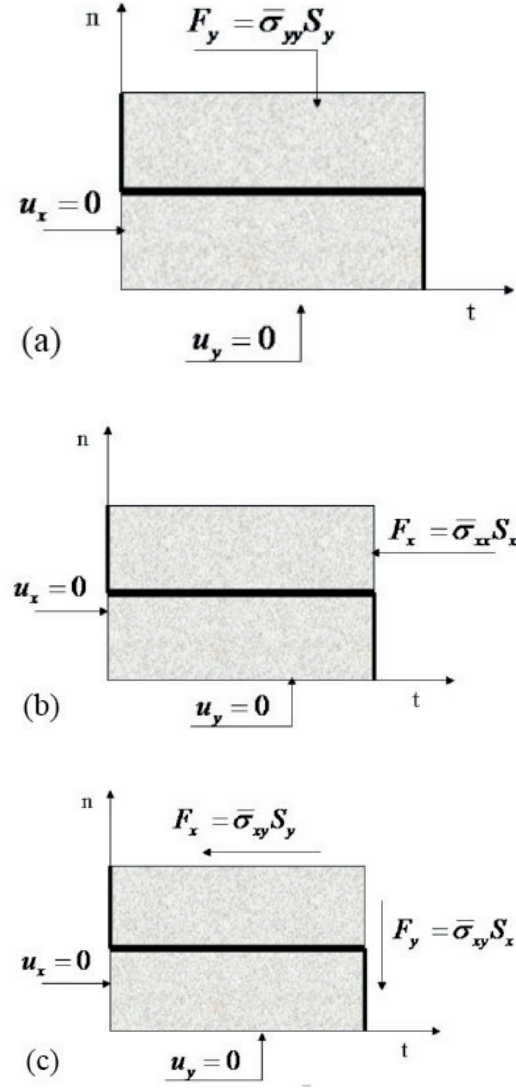

Fig. 15 Boundary and symmetry conditions for the considered quarter cell subjected to axial normal (a) or tangential (b) compression or shear (c) loadings

cell to three types of loadings: axial compression along $n$, axial compression along $t$ and shear loading as shown on Fig. 15). In this case, strain localization components $A_{i j k l}^{r}$ are given by the following equations:

$\bar{\varepsilon}_{x x}^{r}=A_{x x y y}^{r} \bar{\varepsilon}_{y y}, \bar{\varepsilon}_{y y}^{r}=A_{y y y y}^{r} \bar{\varepsilon}_{y y}, \bar{\varepsilon}=\bar{\varepsilon}_{y y} e_{y} \otimes e_{y}$,

$\bar{\varepsilon}_{x x}^{r}=A_{x x x x}^{r} \bar{\varepsilon}_{x x}, \bar{\varepsilon}_{y y}^{r}=A_{y y x x}^{r} \bar{\varepsilon}_{x x}, \bar{\varepsilon}=\bar{\varepsilon}_{x x} e_{x} \otimes e_{x}$,

$\bar{\varepsilon}_{x y}^{r}=2 A_{x y x y}^{r} \bar{\varepsilon}_{x y}, \bar{\varepsilon}=\bar{\varepsilon}_{x y}\left(e_{x} \otimes e_{y}+e_{y} \otimes e_{x}\right)$.

The localization strain tensor $A$ is assumed to be orthotropic. Since the symmetry of the Cauchy strain tensor both in the anisotropic and isotropic spaces is required, it follows that $A_{i j k l}^{r}=A_{j i k l}^{r}=A_{j i l k}^{r}$ (minor symmetry).

The major symmetry of $A^{r}$ is also necessary $A_{i j k l}^{r}=A_{k l j i}^{r}$. Accordingly only the components $A_{x x x x}, A_{y y y y}, A_{x y x y}=A_{x y y x}$ and $A_{y x x y}=A_{y x y x}$ are not null. Owing to the classical Voigt notation, the constitutive behavior law of the unit cell reads

$\left(\begin{array}{c}\bar{\sigma}_{y y} \\ \bar{\sigma}_{x x} \\ \bar{\sigma}_{x y}\end{array}\right)=\left(\begin{array}{ccc}\tilde{C}_{n n n n} & \tilde{C}_{n n t t} & 0 \\ \tilde{C}_{n n t t} & \tilde{C}_{t t t t} & 0 \\ 0 & 0 & \tilde{C}_{n t n t}\end{array}\right)\left(\begin{array}{c}\bar{\varepsilon}_{y y} \\ \bar{\varepsilon}_{x x} \\ 2 \bar{\varepsilon}_{x y}\end{array}\right)$, 
where $\bar{\sigma}=\langle\sigma\rangle_{V}$ is the overall applied stress on the periodic cell. The software Cast3M [54] has been used to provide local mechanical fields and mainly average mechanical fields such as strain $\bar{\varepsilon}^{r}$, stress $\bar{\sigma}^{r}$ over each phase $r(r=b$ for bricks, $m$ for mortar) and macroscopic strain $\bar{\varepsilon}=f_{b} \bar{\varepsilon}^{b}+f_{m} \bar{\varepsilon}^{m}$ calculated in order to deduce components of the effective stiffness tensor $\tilde{C}$ (Eq. (23)). The five engineering "constants" are then given by

$\frac{1}{\tilde{E}_{t t}\left(t, d_{c}\right)}=\frac{\tilde{C}_{n n n n}}{\tilde{C}_{n n n n} \tilde{C}_{t t t t}-\tilde{C}_{t t n n} \tilde{C}_{n n t t}}$,

$\frac{1}{\tilde{E}_{n n}\left(t, d_{c}\right)}=\frac{\tilde{C}_{t t t t}}{\tilde{C}_{n n n n} \tilde{C}_{t t t t}-\tilde{C}_{t t n n} \tilde{C}_{n n t t}}$,

$\tilde{\mu}_{n t}\left(t, d_{c}\right)=\tilde{C}_{n t n t}, \tilde{v}_{n t}\left(t, d_{c}\right)=\frac{\tilde{C}_{n n t t}}{\tilde{C}_{t t t t}}, \tilde{v}_{n t}\left(t, d_{c}\right)=\frac{\tilde{C}_{t t n n}}{\tilde{C}_{n n n n}}$.

Recall that for an isotropic material (brick and mortar), components of the stiffness tensor $C^{r}(r=b, m)$ read (25)

$\tilde{C}_{x x x x}^{r}=\tilde{C}_{y y y y}^{r}=k^{r}+\frac{4}{3} \mu^{r}, \tilde{C}_{x x y y}^{r}=\tilde{C}_{y y x x}^{r}=k^{r}-\frac{2}{3} \mu^{r}, \tilde{C}_{x y x y}^{r}=2 \mu^{r}$ where

$k^{r}=\frac{E^{r}}{3\left(1-2 v^{r}\right)}, \mu^{r}=\frac{E^{r}}{2\left(1+v^{r}\right)}$

are respectively the bulk and shear moduli.

Fig. 16 reports the time evolution of PCW estimates for the masonry's effective Young's $\tilde{E}_{x x}, \tilde{E}_{y y}$ and shear $\tilde{\mu}_{x y}$ moduli. Qualitatively, it can be observed that the Young's moduli decrease quickly during the first 100 days to attain almost their half initial values (at $t=0$ ). Beyond 100 days, these moduli stabilize during a period of about 600 days before decreasing again rapidly beyond $t=800$ (days), but less quickly compared to the first 100 days period. Recall

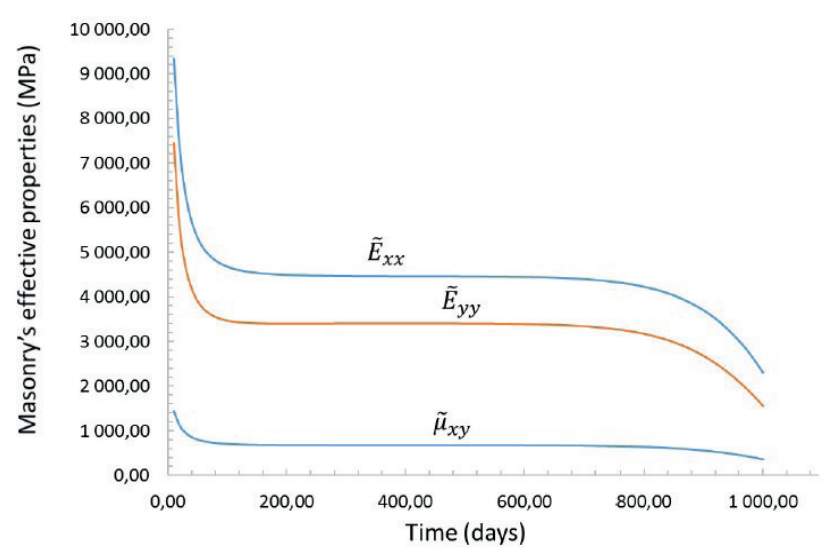

Fig. 16 Times evolutions of the effective properties of a masonry cell constituted by viscous bricks and microcracked mortars joints with open cracks which crack density evolves following a time power-law function that similar trends were observed for dilute estimates of the curves "masonry's effective Young's moduli versus time" where considered bricks were rigid [42]. Effective shear moduli is softer than effective Young's moduli (is about $\tilde{E}_{x x} / 6$, and $\left.\tilde{E}_{y y} / 4\right)$. Moreover, by contrary to Young's moduli, it decreases progressively for $t \leq 100$ days and $t \geq 800$ days (there is no sudden nor intensive decrease). Fig. 17 shows that masonry's macroscopic estimates derived from PCW and dilute schemes are very close for $t \leq 800$ days (error lower than $4 \%$ ).

Beyond this instant the percent error between the two estimates increases rapidly to exceed $10 \%$ and even reach $24 \%$ at $t=1000$ days. At each time t, the $P C W$ estimates are a softer than the dilute ones. On Fig. 18 are depicted time-evolutions of percent error

$$
\operatorname{Err}(\%)=100\left(\frac{A_{v}-A_{e}}{\left(A_{v}+A_{e}\right) / 2}\right),
$$

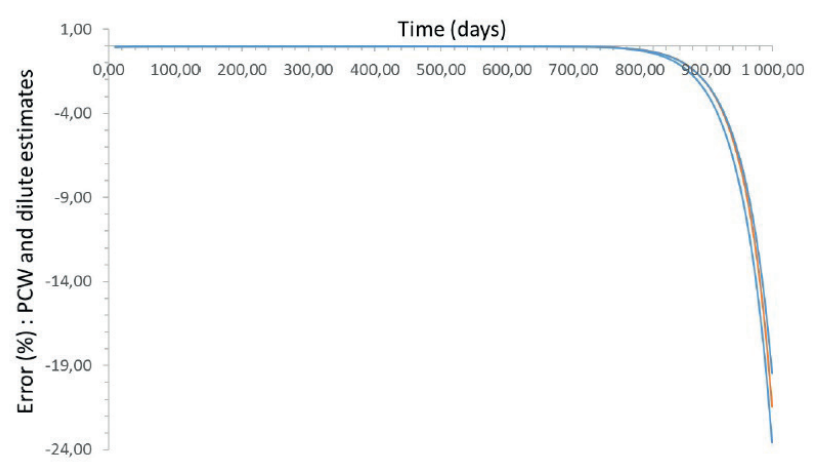

Fig. 17 Comparison between PCW and dilute estimates for effective properties of a masonry cell constituted by viscous bricks and microcracked mortars joints with open cracks which crack density evolves following a time power-law function

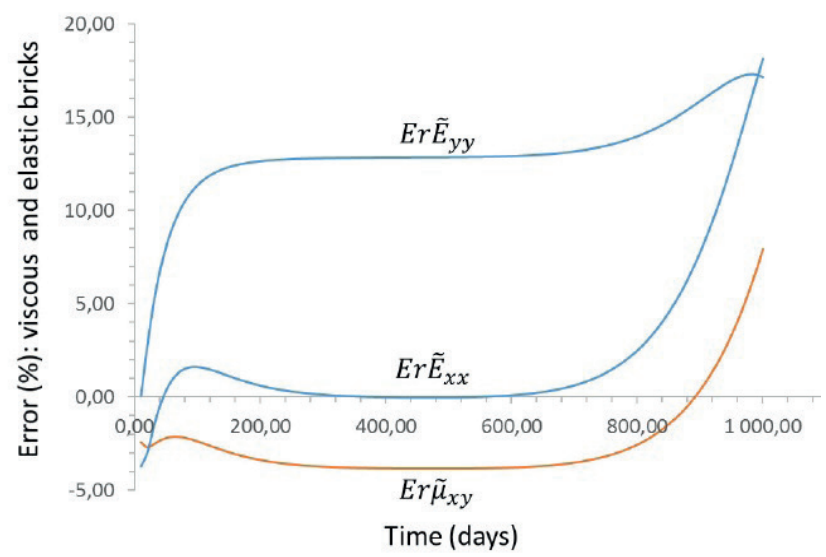

Fig. 18 PCW effective estimates for periodic masonry cell with microcracked mortar joints (open cracks): comparison between elastic and viscous bricks 
between macroscopic properties of masonry with elastic and viscous bricks. The highest gap between the two cases is observed for the $\tilde{E}_{y y}$ modulus. The brick's viscosity increases stiffness along the normal direction $y$. This difference stabilizes around $12 \%$ during a period of about 600 days after a rapid increase during the first 200 days. Beyond $t=800$ days, $\operatorname{Err}(\%)$ increases to exceed $15 \%$. For $\tilde{E}_{x x}$ modulus, differences between the elastic and viscous cases is almost negligible since it is lower than $4 \%$ for $t \leq 800$ days. Similarly to the modulus $\tilde{E}_{y y}$, the error for $\tilde{E}_{x x}$ increases rapidly for $t \geq 800$ days to exceed $15 \%$ at $t=$ 1000 days. By contrary to moduli $\tilde{E}_{x x}$ and $\tilde{E}_{y y}$, shear modulus of masonry with viscous bricks is softer than masonry with elastic bricks. Beyond $t=900$ days, this trend is reversed. However difference between effective modulus $\tilde{\mu}_{x y}$ for the two cases (elastic and viscous) remains acceptable (lower than $8 \%$ for $t \leq 1000$ days).

\section{Local behavior of a masonry wall: open cracks}

Since in the case of closed cracks, the damage is assumed to not evolve [47] and in order to consider interesting problems accounting for crack density evolution, it is proposed in this section to deal only with the case of open cracks.

\subsection{Rigid bricks: comparison between numerical and analytical solutions}

In order to validate the herein proposed numerical model, it is suggested in this part to compare its result in terms of vertical displacement of a compressed wall along the $y$ direction (Fig. 19(a)) to the analytical solution of a homogeneous plate submitted to the same vertical compressive load and assuming the hypothesis of "in-plane stress" [55]. Here, bricks are assumed to be rigid while mortar is viscoelastic following the Generalized Maxwell rheological model which elastic and viscous properties (one term) are provided in Table 1. Accordingly, the mortar's Young's modulus evolves at safe $\left(d_{c}=0\right)$ and micro-cracked $\left(d_{c}\right.$ following time power-law relation (18)) states as reported on Fig. 20. It can be noticed that while error between these states (safe and micro-cracked) is lower than $6 \%$ for time $t \leq 750$ (days), it increases relatively quickly after this limit to reach $22 \%$ at $t=900$ (days).

For a homogeneous vertically compressed plate, we recall that the analytic solution is given by [55]

$u_{y}(x, H, t)=\frac{Q_{1} H}{A_{2222}^{F}(t)(L s)}=\frac{q_{1} H}{A_{2222}^{F}(t)}$

where $L$ and $H$ are respectively the width and height of the panel and $\mathrm{s}$ is the brick thickness. $Q_{1}$ and $q_{1}=Q_{1} /(L s)$ denote respectively the total vertical load and pressure applied at the top of the masonry panel. $A_{i j k l}^{F}$ represent approximate expressions for the homogenized relaxation coefficients provided by the kinematically admissible solution given in [55]. Particularly,

$$
A_{2222}^{F}(t)=\frac{\left(a+e_{h}\right) K_{h}(t)}{e_{h}},
$$

where $a$ denotes the height of the brick, $e_{h}$ is the thickness of the mortar head joints and

$$
K_{h}(t)=\frac{E_{m}(t)}{\left(1+v_{m}\right)\left(1-2 v_{m}\right)}
$$

is the bulk relaxation moduli of horizontal interfaces. In this work, note that viscoelastic behaviors of the bed and head joints (indexed respectively by ' $b$ ' and ' $h$ ') are assumed to be coincident and are defined by the relaxation function $E_{m}(t)$ (instantaneous "Young's modulus" of the mortar given by $E_{m}=1 / J_{M M}^{a p p}$ (see Eq. (15)).

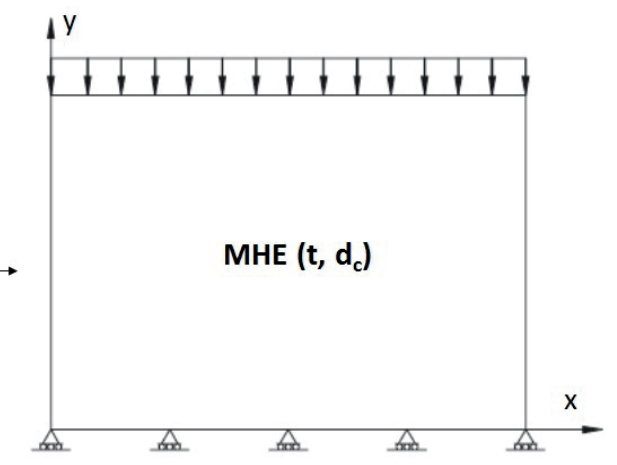

(b)

Fig. 19 Rigid bricks and viscoelastic mortar joints: Real (a) and equivalent homogenized (b) masonry panels subjected to a vertical distributed load applied at the top [56] 


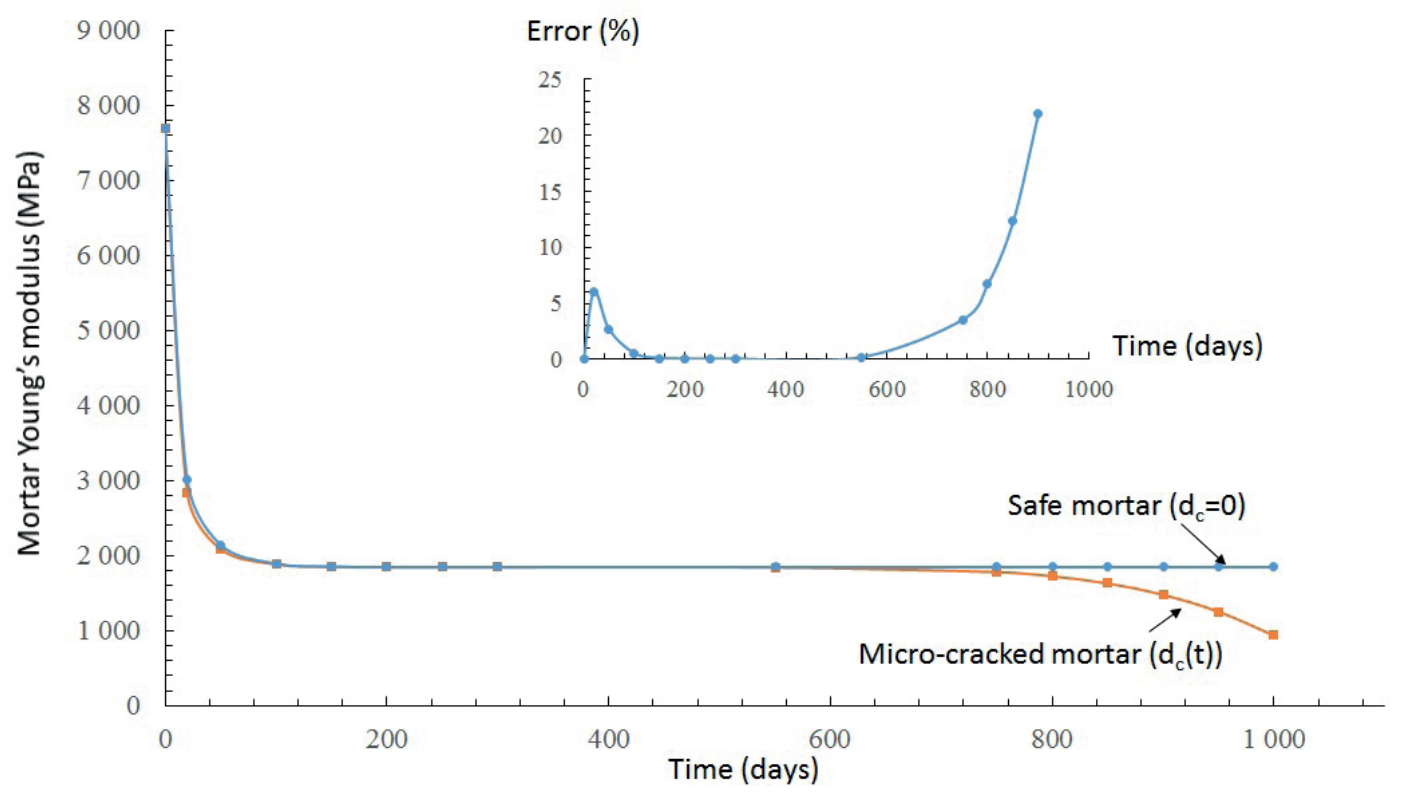

Fig. 20 Mortar joints: Time evolutions of the "instantaneous global Young's moduli Em" of the considered viscoelastic mortar at safe and microcracked states and the error (\%) between these moduli

On the other hand, to obtain the local numerical solution for this structure, the real heterogeneous problem (Fig. 19(a)) is substituted by the homogenized one denoted by $\operatorname{MHE}\left(t, d_{c}\right)$ (Fig. 19(b)) which orthotropic properties used for finite element resolution of the considered problem are determined due to the periodic homogenization procedure previously described in Section 3 .

Fig. 21 reports time evolution of the vertical displacement at the top of the compressed masonry wall composed of rigid bricks and (safe or microcracked) viscoelastic mortar. At the safe state of the mortar joints, this figure allows comparison between finite elements solution and analytical one provided by Eq. (27) assumed to be the reference solution. It can be seen that numeric and analytic solutions show similar qualitative trends. Quantitatively, numeric solution is very close to the analytical one. Indeed, according to Fig. 22, the error between numerical and analytical (red curve) solutions does not exceed $7 \%$ for all time $0 \leq t \leq 1000$ (days). This non-null error (at safe state of viscoelastic masonry) can be explained by the fact that analytic model implies approximate expressions for homogenized relaxation coefficient $\left(A^{F}{ }_{2222}\right)$. Moreover, it is available for masonry with rigid bricks connected by interfaces, however the herein proposed numerical model accounts for finite thickness of mortar joints (here set equal to $10 \mathrm{~mm}$ ).

The curve describing the evolution of the displacement $u_{y}$ at the top of the compressed wall (Fig. 21) with microcracked mortar joints shows qualitative trend similar to that observed for masonry wall with safe mortar joints till

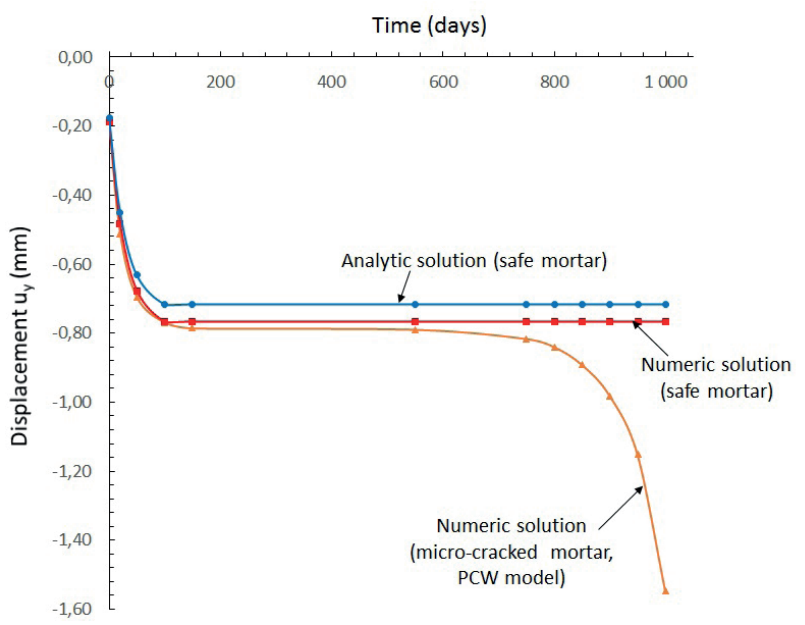

Fig. 21 Rigid bricks and viscoelastic mortar joints: Time evolutions of the local vertical displacement uy at the top of the panel

$t=600$ (days). After that the absolute value of the displacement uy does not stabilize as it increases with time. This is consistent with the decrease of the microcracked mortar's Young's modulus observed on Fig. 20. The difference between safe and micro-cracked states is highlighted by the blue curve depicted on Fig. 22 showing that presence of micro-cracks induces a gap between uy results lower than $13 \%$ for time $t \leq 750$ (days) but which increases with time to reach (for example) $31,3 \%$ at $t=900$ (days). It is worth noting that Fig. 22 reports also curve estimating error between numerical results for uy at the top of the compressed wall with mortar joints at safe and microcracked states. Qualitatively, the trend of this curve is similar to that estimating error between analytic and numeric 
results at safe state of mortar joints. Quantitatively, this curve shows errors lower than those provided by blue curve. Indeed for $t \leq 760$ days, this error is below $7 \%$. After that, it can reach almost $25 \%$ at $t=900$ days which is consistent with the decrease of the microcracked mortar's Young's modulus observed on Fig. 20.

\subsection{Viscoelastic bricks: numerical solution}

This part aims to assess the relevance and efficiency of the proposed model based on the coupling between PCW homogenization model and Griffith's brittle theory. For this purpose, we consider the problem of a viscoelastic masonry wall of dimensions $L=1560 \mathrm{~mm}$ (length) and $H=1040 \mathrm{~mm}$ (height) submitted to compressive loadings (three distributed loads at the top and two lateral edges and an additional concentrated load $\mathrm{F}$ applied on the top) as shown on Fig. 23. Bricks are assumed to be safe and viscous following the $M M$ 's rheological model. The mortar's (respectively, brick's) material data used to compute this problem are given in Table 1 (respectively, Table 4). Crack density is assumed to evolve according to a time power-law function (Eq. (19)). On the other hand, as the arrangement of the bricks is regular, the effective behavior of the panel is assumed to be well estimated by that of a periodic cell which effective behavior (macroscopic effective stiffness) is determined in Section 3. The wall can then be modeled as a homogeneous material which properties coincide with those of the equivalent material HEM-2 (Fig. 1(e)).

On Fig. 24 are depicted the normal and shear stresses distributions in the compressed wall at $t=900$ days. Qualitatively, it can be observed that, under BC-2, distribution of the stress field $\sigma_{y y}$ is symmetric (Fig. 24(a)) by reference to the symmetry axis of the panel $(x=L / 2)$ unlike that of the shear stress $\sigma_{x y}$ which is anti-symmetric (Fig. 24(b)). Similar trends are observed on Fig. 25 related to the case of a wall under compressive loadings without

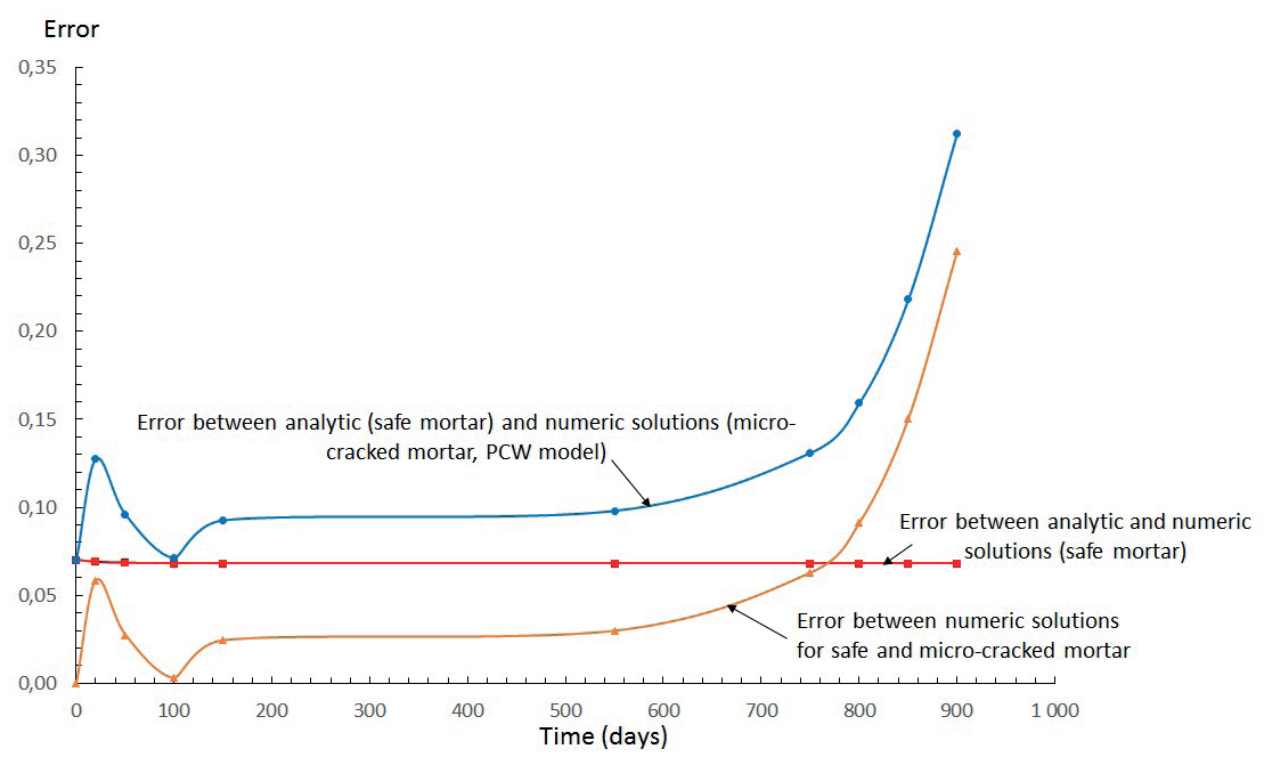

Fig. 22 Time evolutions of the error between analytic (safe mortar) and numerical (safe and microcracked mortar) solutions for vertical displacement uy at the top of the compressed wall
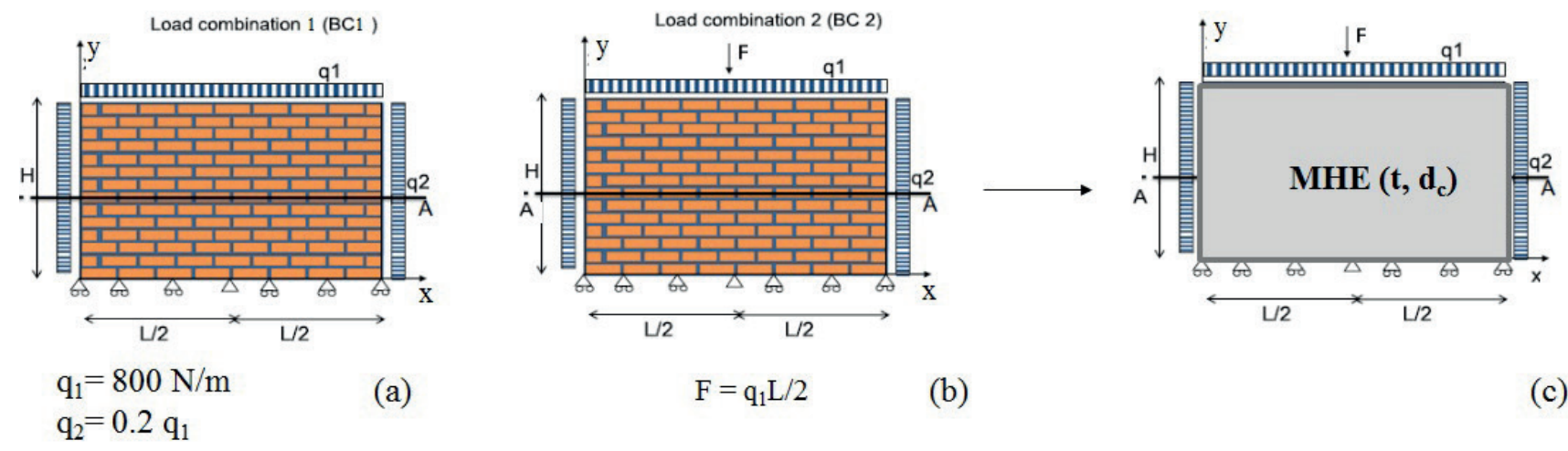

$$
\mathrm{q}_{2}=0.2 \mathrm{q}_{1}
$$

Fig. 23 Masonry panel submitted to boundary conditions BC-1 (a), BC-2 (b) and equivalent problem (c) for masonry under BC-2 
concentrated load $F$ (boundary condition BC-1) showing however different stress localization zones: at the right and left corners of the top of the wall. By contrary, normal and shear stresses concentrate at the vicinity of the application's point of the concentrated load $F$ when considering wall under boundary condition BC-2. Moreover, although under BC-1, compressive loadings induce only compression $\left(\sigma_{y y} \leq 0\right)$ throughout the area of the wall, the additional load $F$ make appears tensile stresses $\left(\sigma_{y y} \geq 0\right)$ in small area surrounding the application point $P$ of the load $F$ as a reaction (response) to the highest values of normal compressive stresses reached around point $P$. Quantitatively, comparison between Figs. 24 and 25 shows that the magnitude of the local normal stress throughout the wall under BC-1 is negligible compared to that attained in a wall under BC-2. Under condition BC-1, it can also be observed that the induced local shear stress is negligible compared to the induced local normal stress. For wall under BC-2, the shear stress is not negligible but (almost 4 times) lower than local normal stress. Fig. 26 illustrating evolutions of stress components at the middle height of the wall $(y=H / 2)$ along the $x$ axis shows difference of stresses magnitudes
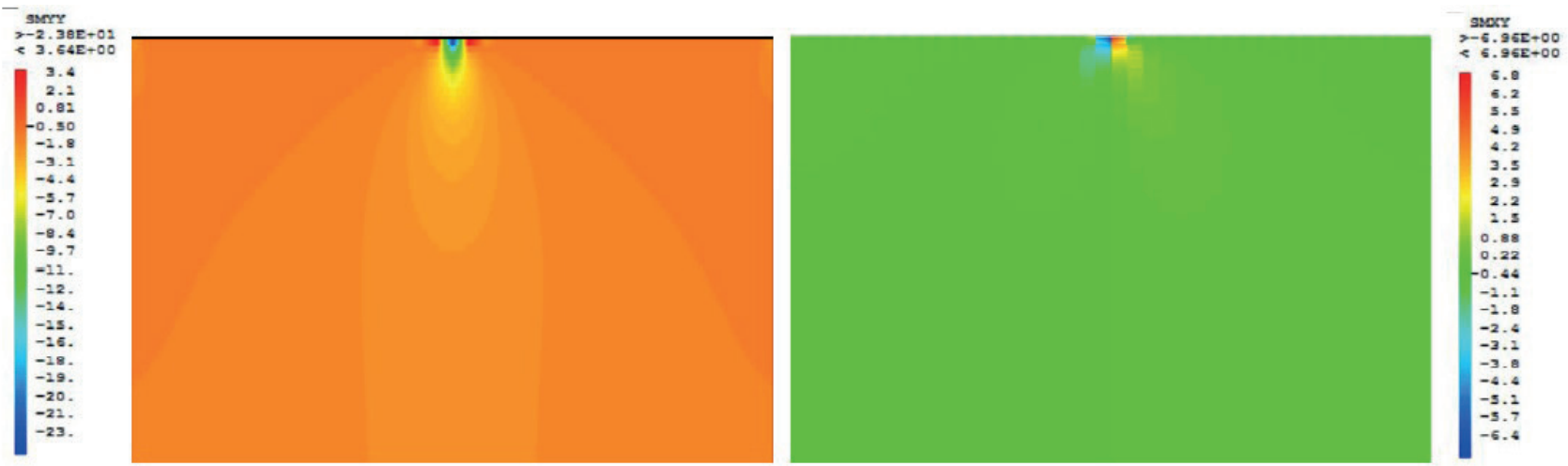

Fig. 24 Local normal (left) and shear (right) stress snapshots throughout a wall (with viscous bricks) under BC-2
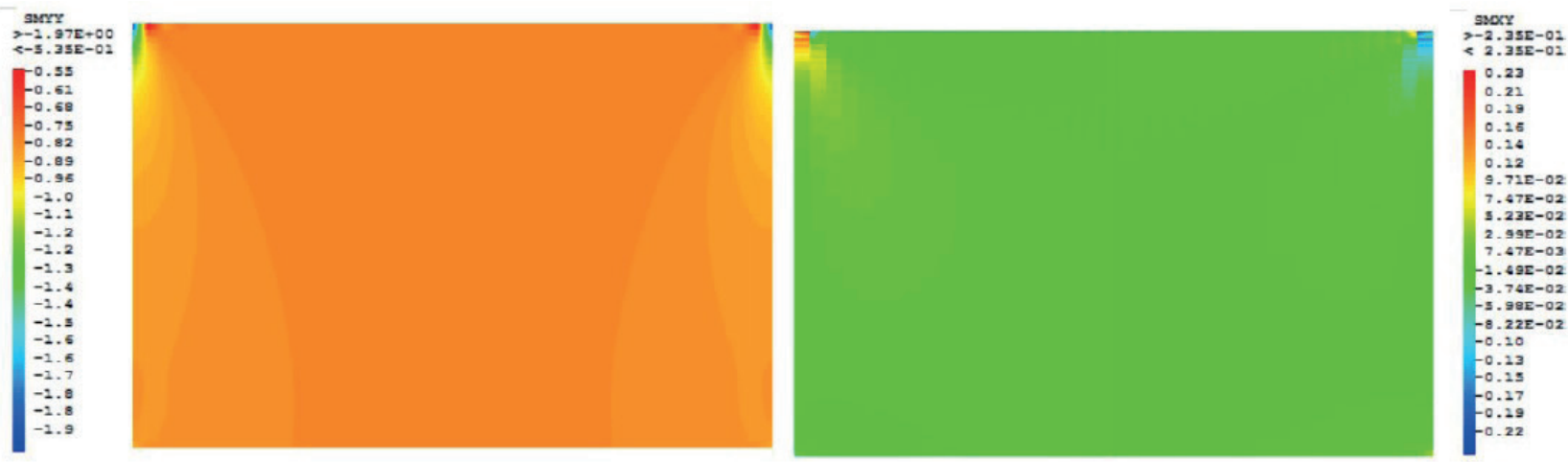

Fig. 25 Local normal (left) and shear (right) stress snapshots throughout a wall (with viscous bricks) under BC-1

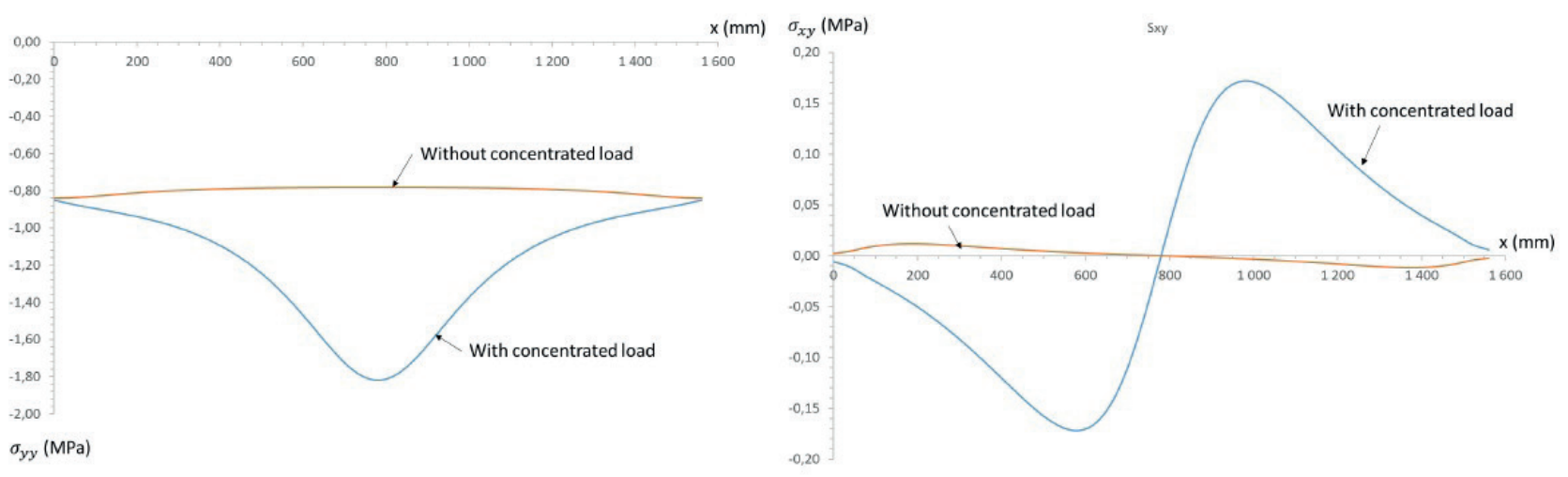

Fig. $26 \mathrm{PCW}$ estimates at time $\mathrm{t}=900$ days for the evolutions of the local stress $\sigma y y$ (left) and $\sigma x y$ (right) versus abciss $\mathrm{x}$ in the middle height of a wall with viscous bricks: comparison between boundary conditions BC-1 and BC-2 
between boundary conditions $\mathrm{BC}-1$ and $\mathrm{BC}-2$ demonstrating that a concentrated load at the middle length of the wall increases the stresses magnitudes and even implies a peak for normal (shear) stress along the axis of the load $F$.

Fig. 27 allows comparison between $P C W$ and dilute estimates for the local normal and shear stresses. For the normal stress, these estimates are close except around the axis of symmetry of the wall where dilute scheme gives softer estimates than $P C W$ model (a percent error of about $8 \%$ is attained at the peak of stress $\sigma_{v y}$ ). This error value is acceptable, however it can be (quite) harmful if we study the risk of failure of the wall. Moreover, this error increases in the vicinity of the application point of the concentrated load (it can exceed $80 \%$ inside the stress localization area). Although Fig. 27(b) shows close estimates deriving from dilute and $P C W$ models, the percent error between these estimates reaches $46 \%$ (Fig. 28) at three zones: near right and left wall edges and area surrounding application point of the concentrated load. This difference is clearly harmful and even dangerous when predicting failure of this compressed wall. The $P C W$ model is then greatly recommended rather than the dilute homogenization model in order to anticipate efficiently the collapse of masonry compressed wall. On the other hand neglecting brick's creep can be acceptable when estimating the normal local stress (see Fig. 29) since error between masonries with elastic and viscous bricks is lower than $4 \%$ at the level of the wall's middle height, however it is strongly harmful for the prediction of magnitudes of shear stresses for which evolution of error along the axis $x$ shows a strong jump at the level of the wall's symmetry axis.

For comparison purpose between strain fields inside masonry under boundary condition BC-1 and BC-2, Fig. 30 reports $\varepsilon_{x y}$ and $\varepsilon_{y y}$ strain evolutions curves versus the $x$ axis. Globally, under these boundary conditions, it is observed that the $M M$ 's model predicts small strains. Moreover, qualitatively, these evolutions show similar trends (symmetric and anti-symmetric evolutions by reference to the axis $x=L / 2$ respectively for $\varepsilon_{y y}$ and $\varepsilon_{x y}$ ) as those observed for the normal and shear stress. Indeed, under $\mathrm{BC}-1$, normal strain is almost constant with variation of the abciss $x$.

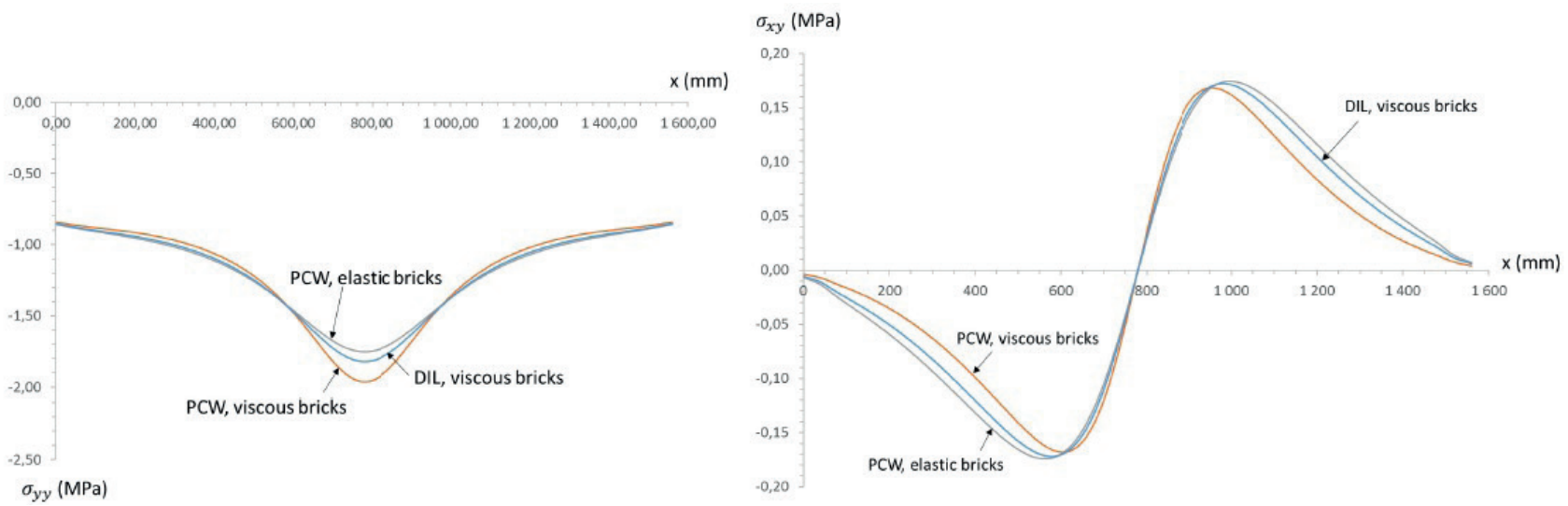

Fig. 27 Boundary condition BC-2: comparison between PCW and dilute estimates for the evolutions of normal and shear stresses versus x axis (wall with viscous bricks) and study of the effect of accounting for creep of bricks
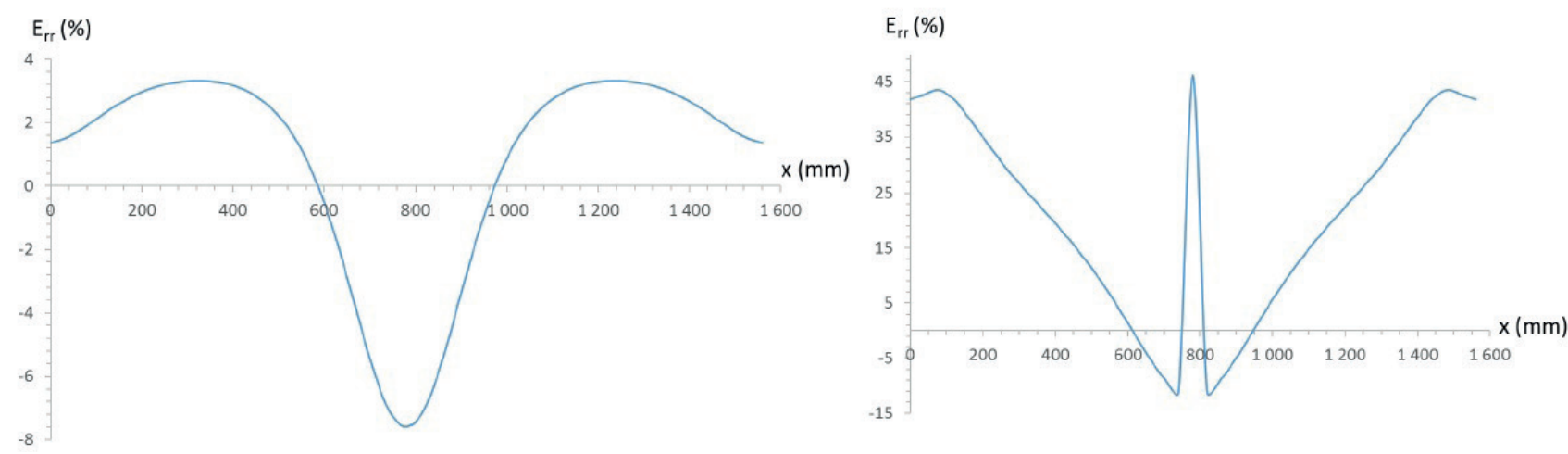

Fig. 28 Percent error between dilute and PCW estimates for local normal (left) and shear (right) stresses in the middle height of the wall (case with viscous bricks) 

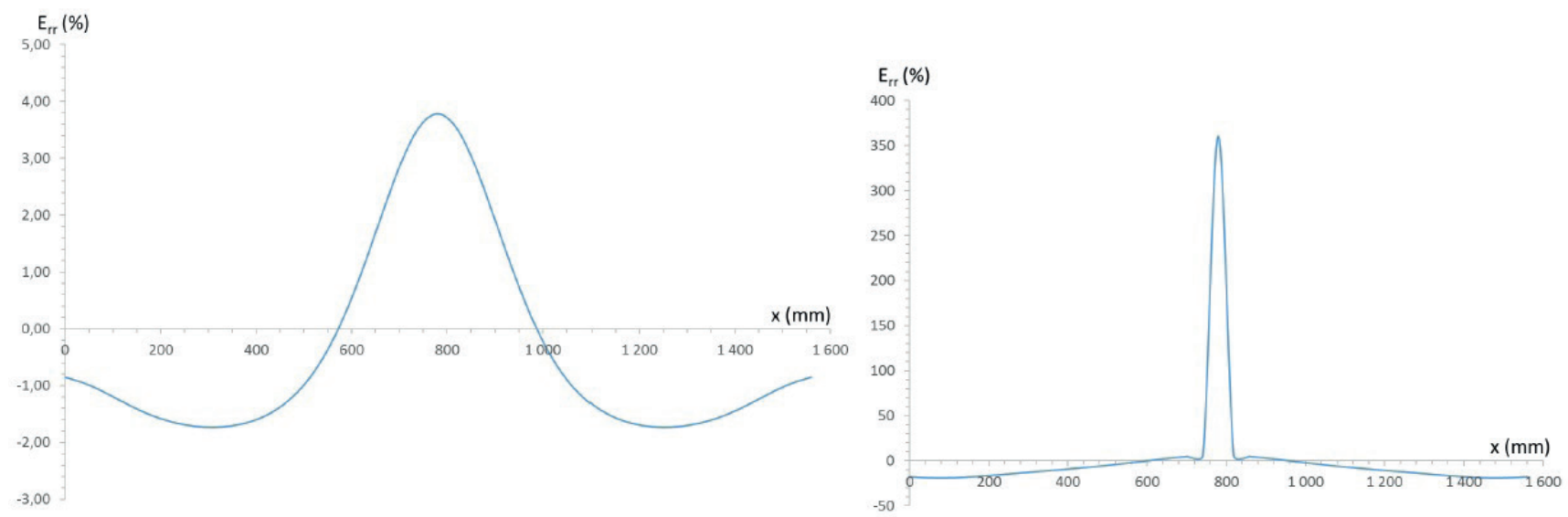

Fig. 29 Percent error between PCW estimates for local normal (left) and shear (right) stresses in the middle height of the wall: effect of bricks' creep
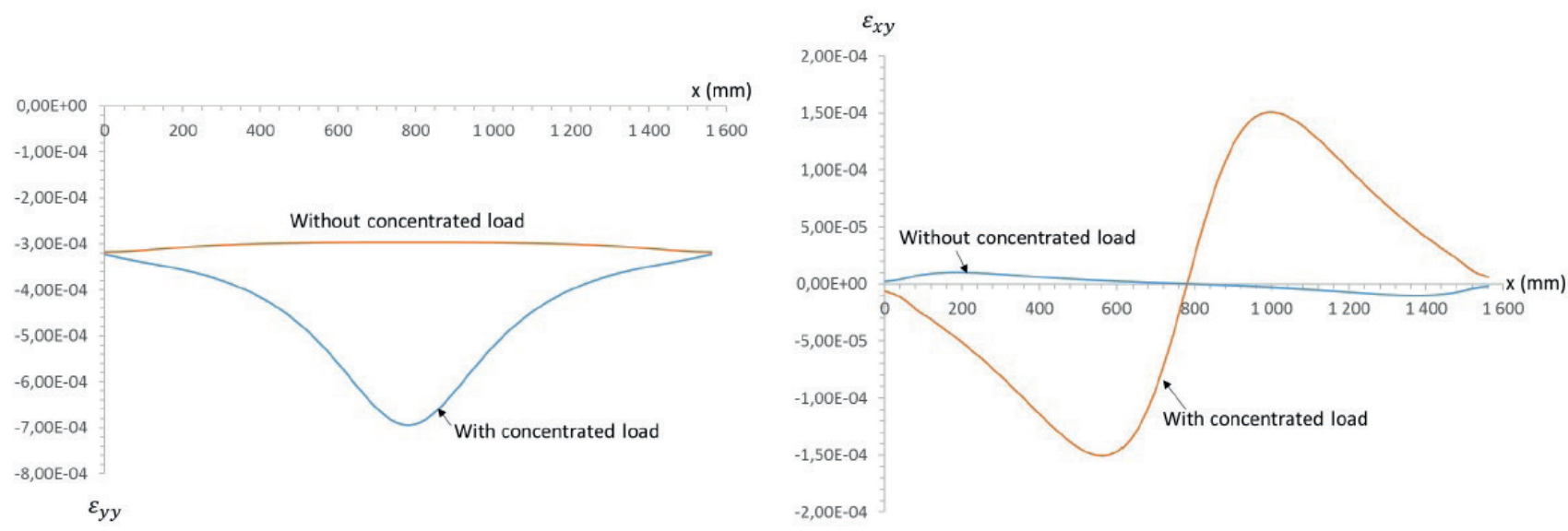

Fig. $30 \mathrm{PCW}$ estimates at time $t=900$ days for the evolutions of the local strain $\varepsilon_{y y}$ (left) and $\varepsilon_{x y}$ (right) versus abciss $\mathrm{x}$ in the middle height of a wall with viscous bricks: comparison between boundary conditions $\mathrm{BC}-1$ and $\mathrm{BC}-2$

However absolute value of the strain $\varepsilon_{y y}$ presents a peak around the symmetry axis of the wall. The magnitude of this peak is two times higher than values of normal strain attained at the level of the wall's edges. Clearly, normal strains induced by condition $\mathrm{BC}-2$ are higher than those attained under condition $\mathrm{BC}-1$ due to the additional concentrated load $F$. This remark remains available for the shear strain predicted at the wall line $y=H / 2$. Sign of corresponding shear peaks under $\mathrm{BC}-1$ and $\mathrm{BC}-2$ are opposite.

For masonry with viscous bricks, comparison between dilute and PCW estimates for the local normal (shear) strain field as depicted on Fig. 31(a) (respectively 31(b)) shows that the dilute scheme highly underestimates (in terms of absolute value) the $P C W$ predictions (considered as a reference local solution) for all abciss $x \in[0, L]$. Errors between PCW and dilute estimates for normal and shear strains are significant. For the former, this error varies between $48 \%$ and $59.3 \%$. for shear strain $\operatorname{Err}(\%)$ belongs to the interval [10\%, $66.9 \%]$. Similarly to the shear stress, the maximal error value for shear strain is attained around the symmetry axis of the wall $(x=L / 2)$. This result confirms again that neglecting cracks interactions highly underestimates stress and strain local (normal and shear) fields throughout the compressed wall. This could lead to false interpretations of the magnitude of local stress and strain fields throughout the studied wall and accordingly to not properly anticipate the wall's failure. At last, comparison between $P C W$ predictions for the strain fields for masonries with elastic and viscous bricks (Fig. 32) reveals that absolute normal strain values attained inside the masonry with elastic bricks are higher than masonry with viscous bricks. In this context, errors for normal strain varies between $12.4 \%$ and $17.8 \%$. However evolution of error related to shear strain show a strong jump around the symmetry axis of the wall. Otherwise it varies between about $4.3 \%$ and $18.1 \%$

\section{Synthesis of the results}

In the following, Tables 5 and 6 summarize trends of above obtained results at three levels: micro-cracked mortar, masonry periodic cell and compressed wall. 

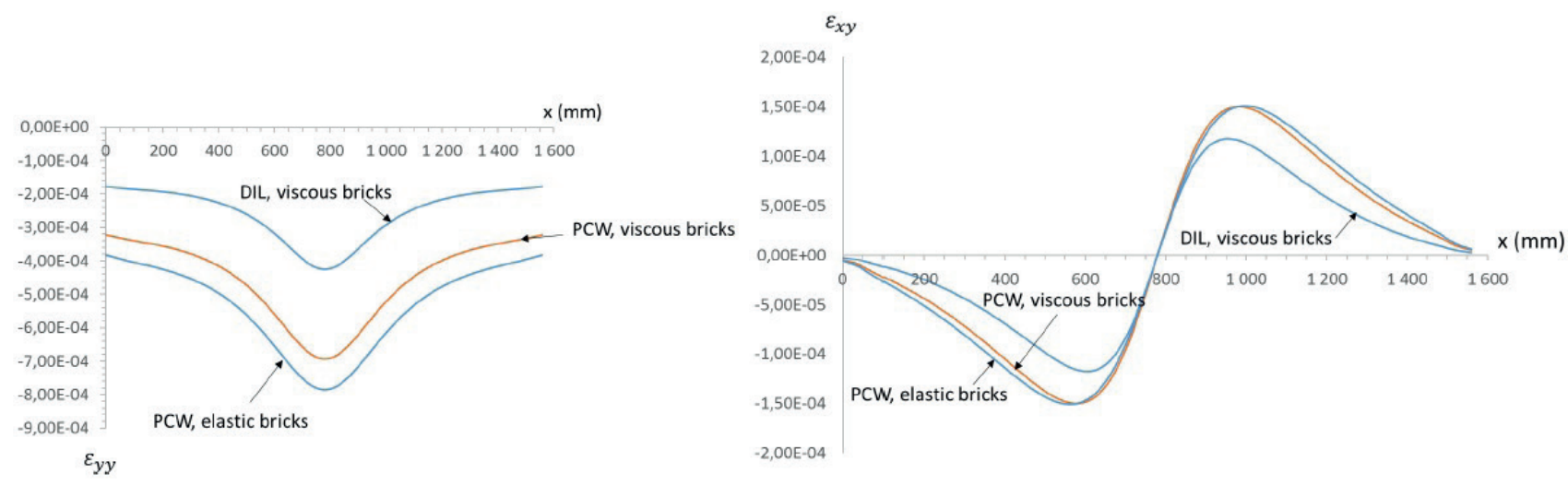

Fig. 31 Boundary condition BC-2: comparison between PCW and dilute estimates for the evolutions of normal and shear stresses versus $x$ axis (wall with viscous bricks) and study of the effect of accounting for creep of bricks
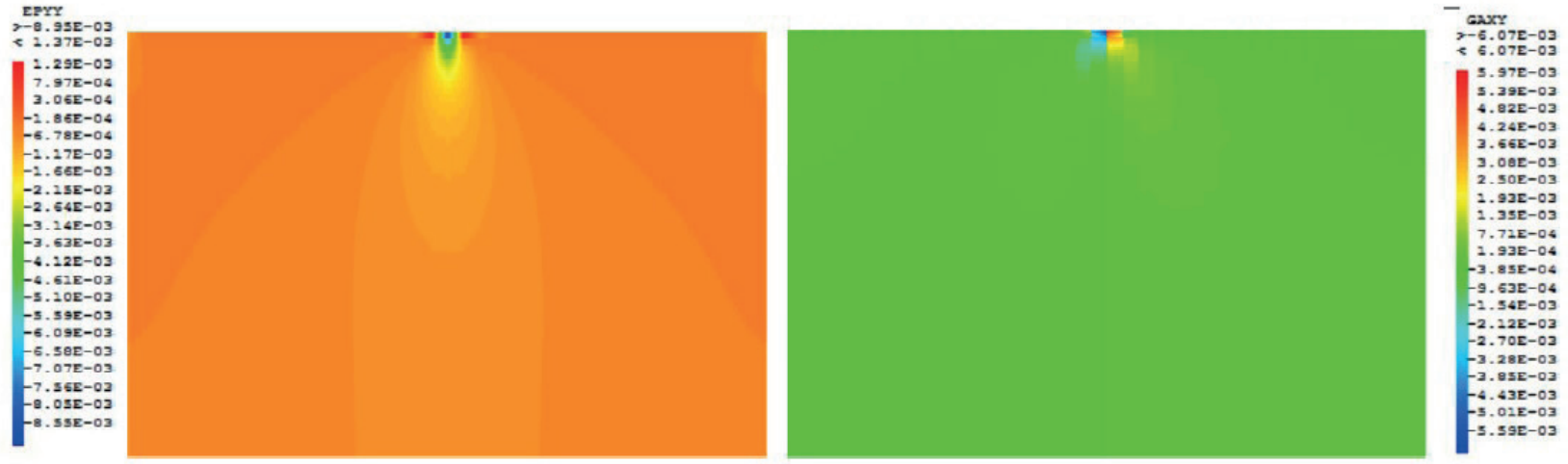

Fig. 32 Local normal (left) and shear (right) strain snapshots throughout a wall (with viscous bricks) under BC-2

Table 5 Comparison between PCW and dilute estimates at three levels: microcracked mortar, masonry periodic cell and compressed wall

Trends

Mortar's effective Young's modulus

DIL $>$ PCW, $E_{r r} \geq 10 \%$, for $d_{c} \geq 0.25$, if open cracks ( 0.55 if closed cracks).

Masonry's effective cell properties (open cracks $\& d_{c}=$ time power-law function) $\mathrm{DIL} \geq \mathrm{PCW}\left(E_{r r} \rightarrow 19\right.$ to $24 \%$ for $\left.d_{c} \rightarrow 0.33\right)$

Local fields in a compressed wall (open cracks $\& d_{c}=$ time power-law function)

$$
\begin{gathered}
\left.\left|\sigma_{y y}^{D I L}\right| \text { and }\left|\sigma_{y y}^{P C W}\right| \text { are close }\left(-8 \% \leq E_{r r} \leq 4 \%\right]\right) \\
\left(\left|\sigma_{y y}^{D I L}\right| \text { slightly stiffer than }\left|\sigma_{y y}^{P C W}\right|\right) \\
\left|\sigma_{x y}^{D I L}\right| \ll\left|\sigma_{x y}^{P C W}\right|(\operatorname{Err} \in[-15 \%, 45 \%]) \\
\left|\varepsilon_{y y}^{D I L}\right| \ll\left|\varepsilon_{y y}^{P C W}\right|(\operatorname{Err} \in[47 \%, 60 \%]) \\
\left|\varepsilon_{x y}^{D I L}\right| \ll\left|\varepsilon_{x y}^{P C W}\right|(\operatorname{Err} \in[10 \%, 70 \%])
\end{gathered}
$$

\section{Conclusions and perspectives}

This work provides a multi-scale improved model to the earlier one proposed in [43, 42] allowing rigorous assessment at short and long-terms of global and local behavior of microcracked viscoelastic masonry. Advantages of the proposed model are mainly related to the none recourse to numerical inversion of the Laplace-Carson transform
$[57,58]$ when assessing the overall creep behavior of mortar and periodic masonry cell, to the ability of this model to take into account cracks interactions, creep of bricks units and variation of mortar's "Poisson's ratio" with crack density. Assuming that the PCW estimates are the reference solution at local and global levels, this work shows that the dilute scheme overestimates the micro-cracked 
Table 6 Comparison between masonry with elastic and viscous bricks: PCW estimates

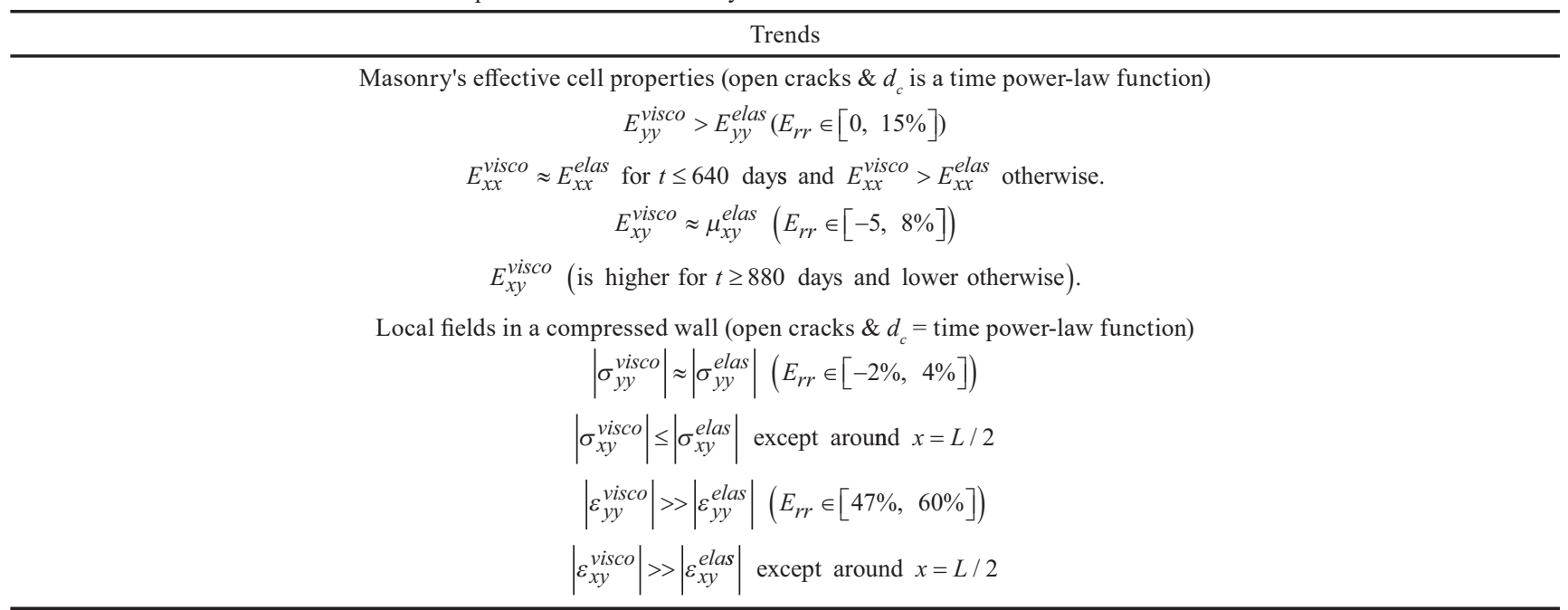

mortar's overall properties whether with open or closed cracks. This result is available at the level of the masonry's periodic cell (with open cracks which crack density evolves owing to a time power-law function) in terms of overall orthotropic properties. At the level of a compressed masonry wall with a concentrated load exerted on its top, it has been observed that dilute estimates for local shear stresses highly underestimate the PCW ones. Moreover, even if percent error for dilute and PCW estimates for the local normal stress can be acceptable at the middle height of the wall, it can be significant in the wall area located at the vicinity of application point of the concentrated load. These results demonstrates that using the dilute scheme instead of the PCW model to predict the local behavior of a compressed wall can be harmful when investigating the risk of failure. This work shows also that viscosity of bricks increases the overall stiffness of masonry cell (of about $15 \%$ to $17 \%$ for $\tilde{E}_{y y}$ and $8 \%$ for $\tilde{\mu}_{x y}$ if $\left.d_{c}=0.33\right)$ for none negligible crack density $\left(d_{c} \geq 0.12\right)$ of microcracks inside mortar joints. This effect is more significant along the direction of head joints even if $d_{c}$ is small $\left(0 \leq d_{c} \leq 0.12\right)$. At the level of the investigated compressed wall, creep of bricks induces higher strains but lower (normal and shear) stresses except around the axis of

\section{References}

[1] Alpa, G., Monetto, I. "Microstructural model for dry block masonry walls with in-plane loading", 42(7), pp. 1159-1175, 1994. https://doi.org/10.1016/0022-5096(94)90065-5

[2] Angelillo, M. "Constitutive relations for no-tension materials", 28, pp. 195-202, 1993.

https://link.springer.com/article/10.1007/BF00989121 symmetry of the wall (direction of the concentrated load) where relative error between local normal (shear) stresses in masonry with elastic and viscous bricks attain its peak. This error is (highly) more significant for local shear stresses at the vicinity of wall edges (of about $20 \%$ ) and mainly around the axis of the concentrated load $(x=L / 2)$. Moreover this demonstrates the fact that neglecting the creep of bricks could false prediction of failure in the wall.

In this work, for the sake of simplicity and trends, the crack density is assumed to follow a time power-law function [52]. In the future, it will be interesting to investigate damage propagation in a thermodynamics framework [48] or by a stress criterion [59]. For bricks and mortar, considering creep functions following the Generalized Maxwell (GM) model with two terms could enrich and increase the accuracy of predictions provided by the proposed model. Moreover, it could be interesting to assess the relevance of the proposed model by comparison with a "full" heterogeneous finite elements model (by modelling all bricks and microcracked viscoelastic mortar joints). At last, accounting for thermal loading could extend this model for refractory linings present for example in blast furnaces subjected to compressive loads resulting from constrained thermal expansion $[60,61]$.

[3] Andreev, K., Sinnema, S., Rekik, A., Allaoui, S., Blond, E., Gasser, A. "Compressive behaviour of dry joints in refractory ceramic masonry", Construction and Building Materials, 34, pp. 402-408, 2012.

https://doi.org/10.1016/j.conbuildmat.2012.02.024 
[4] Anthoine, A. "Derivation of in-plane elastic characteristics of masonry through homogenization theory", International Journal of Solids and Structures, 32(2), pp. 137-163, 1995. https://doi.org/10.1016/0020-7683(94)00140-R

[5] Baraldi, D., Cecchi, A. "Discrete element model for in-plane loaded viscoelastic masonry", Journal for Multiscale Computational Engineering, 12(2), pp. 155-175, 2014. https://doi.org/10.1615/IntJMultCompEng.2014008118

[6] Binda, L., Gatti, G., Mangano, G., Poggi, C., Sacchi Landriani, G. "The Collapse of Civic Tower of Pavia: A Survey of the Materials and Structure", Masonry International, 6(1), pp. 11-20, 1992. [online] Available at: https://www.masonry.org.uk/downloads/thecollapse-of-the-civic-tower-of-pavia-a-survey-of-the-materialsand-structure/ [Accessed: 8 February 2020]

[7] Bornert, M., Bretheau, T., Gilormini, P. "Homogénéisation en mécanique des matériaux, Tome 1 : Matériaux aléatoires élastiques et milieux périodiques" (Homogenization in mechanics of materials 1: elastic random materials and periodic media), Hermes Science, Paris, France, 2001. [online] Available at: https://www.lavoisier.fr/livre/ materiaux/homogeneisation-en-mecanique-des-materiaux-1-materiaux-aleatoires-elastiques-et-milieux-periodiques/bornert/ descriptif-9782746201996 [Accessed: 8 February 2020] (in French)

[8] Briccola, D., Bruggi, M. "Analysis of 3D linear elastic masonry-like structures through the API of a finite element software", Advances in Engineering Software, 133, pp. 60-75, 2019.

https://doi.org/10.1016/j.advengsoft.2019.04.009

[9] Briccola, D., Bruggi, M., Taliercio, A. "Analysis of 3D no-tension masonry-like walls", Journal of Mechanics of Materials and Structures, 13(5), pp. 631-646, 2018.

https://doi.org/10.2140/jomms.2018.13.631

[10] Brooks, J. J. "Composite modeling of masonry deformation", Materials \& Structures, 23, pp. 241-251, 1990. https://doi.org/10.1007/BF02472197

[11] Bruggi, M., Taliercio, A. "Analysis of no-tension structures under monotonic loading through an energy-based method", Computers \& Structures, pp. 159, 14-25, 2015.

https://doi.org/10.1016/j.compstruc.2015.07.002

[12] Budiansky, B., O'Connell, R. "Elastic moduli of a cracked solid", International Journal of Solids Structures, 12(2), pp. 81-97, 1976. https://doi.org/10.1016/0020-7683(76)90044-5

[13] Huy Duong, B., Germain, P. "Mécanique de la rupture fragile" (Fragile fracture mechanics), Masson, Paris, France, 1978. (in French)

[14] University of Montpellier "Cast3M" (online) Available at: http:// www-cast3m.cea.fr/ [Accessed: 8 February 2020]

[15] Ponte Castañeda, P., Willis, J. R. "The effect of spatial distribution on the effective behavior of composite materials and cracked media", Journal of the Mechanics and Physics of Solids, 43(12), pp. 1919-1951, 1995. https://doi.org/10.1016/0022-5096(95)00058-Q

[16] Cecchi, A., Sab, K. "A comparison between a 3D discrete model and two homogenised plate models for periodic elastic brickwork", International Journal of Solids Structures, 41(9-10), pp. 22592276, 2004.

https://doi.org/10.1016/j.ijsolstr.2003.12.020
[17] Cecchi, A., Taliercio, A. "A comparison between numerical and analytical homogenized models for visco-elastic brickwork", presented at XXI. Congresso Dell'Associazione Italiana di Meccanica Teorica e Applicata - AIMETA 2013, Torino, Italy, Sept, 17-20, 2013. [online] Available at: http://hdl.handle.net/11311/762126 [Accessed: 8 February 2020] (in Italian)

[18] Cecchi, A., Tralli, A. "A homogenized viscoelastic model for masonry structures", International Journal of Solids and Structures, 49(13), pp. 1485-1496, 2012. https://doi.org/10.1016/j.ijsolstr.2012.02.034

[19] Wu, C., Hao, H. "Derivation of 3D masonry properties using numerical homogenization technique", International Journal For Numerical Methods in Engineering, 66(11), pp. 1717-1737, 2006. https://doi.org/10.1002/nme.1537

[20] Choi, K.-K., Lissel, S. L., Reda Taha, M. M. "Rheological modelling of masonry creep", Canadian Journal of Civil Engineering, 34(11), pp. 1506-1517, 2007. https://doi.org/10.1139/L07-062

[21] Como, M. "Statics of Historic Masonry Constructions", Springer, Berlin, Heidelberg, Germany, 2017. https://oi.org/10.1007/978-3-642-30132-2

[22] Cost, T. L., Becker, E. B. "A multidata method of approximate laplace transform inversion", International Journal for Numerical Methods in Engineering, 2(2), pp. 207-219, 1970. https://doi.org/10.1002/nme.1620020206

[23] Cluni, F., Gusella, V. "Homogenisation of non-periodic masonry structures", International Journal of Solids and Structures, 41(7), pp. 1911-1923, 2004. https://doi.org/10.1016/j.ijsolstr.2003.11.011

[24] de Buhan, P., de Felice, G. "A homogenisation approach to the ultimate strength of brick masonry", Journal of the Mechanics and Physics of Solids, 45(7), pp. 1085-1104, 1997. https://doi.org/10.1016/S0022-5096(97)00002-1

[25] Deudé, V., Dormieux, L., Kondo, D., Pensée, V. "Propriétés élastiques non linéaires d'un milieu mésofissuré" (Nonlinear elastic properties of a mesocracked medium), Comptes Rendus Mécanique, 330(8), pp. 587-592, 2002. https://doi.org/10.1016/S1631-0721(02)01489-4

[26] Dormieux, L., Kondo, D. "Stress-based estimates and bounds of effective elastic properties: the case of cracked media with unilateral effects", Computational Materials Science, 46(1), pp. 173179, 2009. https://doi.org/10.1016/j.commatsci.2009.02.027

[27] Dormieux, L., Kondo, D., Ulm, F.-J. "Microporomechanics", John Wiley \& Sons, Chichester, UK, 2006.

[28] Di Pasquale, S. "New trends in the analysis of masonry structures", Meccanica, 27, pp. 173-84, 1992. https://doi.org/10.1007/BF00430043

[29] Ferber, M. K., Weresczak, A. A., Hemrick, J. G. "Compressive Creep and Thermophysical Performance of Refractory Materials", Oak Ridge National Laboratory, Oak Ridge, TN, USA, Rep. ORNL/ TM-2005/134, 2006. https://doi.org/10.2172/885151 
[30] Gabor, A., Ferrier, E., Jacquelin, E., Hamelin, P. "Analysis of the inplane shear behaviour of FRP reinforced hollow brick masonry walls", Structural Engineering and Mechanics, 19(3), pp. 237-260, 2005.

https://doi.org/10.12989/sem.2005.19.3.237

[31] Gabor, A., Bennani, A., Jacquelin, E., Lebon, F. "Modelling approaches of the in-plane shear behaviour of unreinforced and FRP strengthened masonry panels", Computers and Structures, 74(3), pp. 277-288, 2006.

https://doi.org/10.1016/j.compstruct.2005.04.012

[32] Giambanco, G., Di Gati, L. "A cohesive interface model for the structural mechanics of block masonry", Mechanica Research Communications, 24(5), pp. 503-512, 1997. https://doi.org/10.1016/S0093-6413(97)00055-4

[33] Hill, R. "Elastic properties of reinforced solids: Some theoretical principles", Journal of the Mechanics and Physics of Solids 11(5), pp. 357-372, 1963. https://doi.org/10.1016/0022-5096(63)90036-X

[34] Ignoul, S., Schueremans, L., Tack, J., Swinnen, L., Feytons, S., Binda, L. A., Van Gemert, D. A., Van Balen, K. V. "Creep behaviour of masonry structures - failure prediction based on a rheological model and laboratory tests", In: Lourenço, P. B., Roca, P., Modena, C., Agrawal, S. (eds.) Proceeding of the 5th International Conference of Structural Analysis of Historical Constructions, New Delhi, India, Nov, 6-8, 2007.

[35] Lourenço, P. B., Rots, J. G. "A multisurface Interface Model for the Analysis of Masonry Structures", Journal of Engineering Mechanics, 123(7), pp. 660-668, 1997. https://doi.org/10.1061/(ASCE)0733-9399(1997)123:7(660)

[36] Lotfi, H. R., Shing, P. B. "Interface Model Applied to Fracture of Masonry Structures", Journal of Structural Engineering ASCE, 120(1), pp. 63-80, 1994. https://doi.org/10.1061/(ASCE)0733-9445(1994)120:1(63)

[37] Lourenço, P. B., Rots, J. G., Blaauwendraad, J. "Continuum Model for Masonry: Parameter Estimation and Validation", Journal of Structural Engineering, 124(6), pp. 642-652, 1998. https://doi.org/10.1061/(ASCE)0733-9445(1998)124:6(642)

[38] Luciano, R., Sacco, E. "Homogenisation technique and damage model for old masonry material", International Journal of Solids and Structures, 34(24), pp. 3191-3208, 1997. https://doi.org/10.1016/S0020-7683(96)00167-9

[39] Marfia, S., Sacco, E. "Numerical Procedure for Elasto-Plastic NoTension Model", International Journal of Computational Methods in Engineering Science and Mechanics, 6(3), pp. 187-199, 2005. https://doi.org/10.1080/15502280590923739

[40] Masiani, R., Trovalusci, P. "Cosserat and Cauchy materials as continuum models of brick masonry", Meccanica, 31, pp. 421-432, 1996. https://doi.org/10.1007/BF00429930

[41] Massart, T. J., Peerlings, R. H. J., Geers, M. G. D. "Structural Damage Analysis of Masonry Walls using Computational Homogenization", International Journal of Damage Mechanics, 16(2), pp. 199-226, 2007. https://doi.org/10.1177/1056789506064943
[42] Nedjar, B., Le Roy, R. "An approach to the modeling of viscoelastic damage: application to the long-term creep of gypsum rock materials", International Journal for Numerical and Analytical Methods in Geomechanics, 37(9), pp. 1066-1078, 2013. https://doi.org/10.1002/nag.1138

[43] Nguyen, S. T. "Propagation de fissures et endommagement par microfissures dans un milieu viscoélastique linéaire non vieillissant" (Propagation of cracks and damage by microcracks in a non-aging linear viscoelastic medium), PhD Thesis, University Paris-Est, 2010. (in French)

https://tel.archives-ouvertes.fr/tel-00598511

[44] Nguyen, T. T. N. "Approche multi-échelles pour des maçonneries viscoélastiques", PhD Thesis, University of Orléans, 2015. (online) Available at: http://www.theses.fr/2015ORLE2077 [Accessed: 8 February 2020] (in French)

[45] Nguyen, S. T., Dormieux, L., Le Pape, Y., Sanahuja, J. "Crack propagation in viscoelastic structures: theoretical and numerical analyses", Computational Materials Science, 50(1), pp. 83-91, 2010. https://doi.org/10.1016/j.commatsci.2010.07.010

[46] Nguyen, S. T., Dormieux, L., Le Pape, Y., Sanahuja, J. "A Burger Model for the Effective Behavior of a Microcracked Viscoelastic Solid", International Journal of Damage Mechanics, 20(8), pp. 1116-1129, 2011 https://doi.org/10.1177/1056789510395554

[47] Taliercio, A., Papa, E. "Modeling of the long-term behaviour of historical masonry towers", In: Binda, L. (ed.) Learning from Failure: Long-term Behaviour of Heavy Masonry Structures, WIT Press, Southampton, UK, 2008, pp. 153-173.

[48] Pelissou, C., Lebon, F. "Asymptotic modeling of quasi-brittle interfaces", Computers and Structures, 87(19-20), pp. 1216-1223, 2009. https://doi.org/10.1016/j.compstruc.2008.12.002

[49] Pegon, P., Pinto, A. V., Géradin, M. "Numerical modeling of stoneblock monumental structures", Computers and Structures, 79(2225), pp. 2165-2181, 2001. https://doi.org/10.1016/S0045-7949(01)00070-0

[50] Pegon, P., Anthoine, A. "Numerical strategies for solving continuum damage problems with softening: application to the homogenization of masonry", Computers and Structures, 64(1-4), pp. 623-642, 1997.

https://doi.org/10.1016/S0045-7949(96)00153-8

[51] Rekik, A., Gasser, A. "Numerical homogenization model for effective creep properties of microcracked masonry", International Journal of Solids and Structures, 102-103, pp. 297-320, 2016. https://doi.org/10.1016/j.ijsolstr.2016.09.028

[52] Rekik, A., Nguyen, T. T. N., Gasser, A. "Multi-level modeling of microcracked viscoelastic masonry", International Journal of Solids and Structures, 81, pp. 63-83, 2016. https://doi.org/10.1016/j.ijsolstr.2015.11.002

[53] Rekik, A., Brenner, R. "Optimization of the collocation inversion method for the linear viscoelastic homogenization", Mechanics Research Communications, 38(4), pp. 305-308, 2011. https://doi.org/10.1016/j.mechrescom.2011.04.003

[54] Rekik, A., Lebon, F. "Homogenization methods for interface modeling in damaged masonry", Advances in Engineering Software, 46(1), pp. 35-42, 2012.

https://doi.org/10.1016/j.advengsoft.2010.09.009 
[55] Sayed-Ahmed, E. Y., Shrive, N. G., Tilleman, D. "Creep deformation of clay masonry structures: a parametric study", Canadian Journal of Civil Engineering, 25(1), pp. 67-80, 1998. https://doi.org/10.1139/197-026

[56] Schapery, R. A. "Approximate methods of transform inversion for viscoelastic stress analysis", In: Proceedings of the fourth U.S. National Congress of Applied Mechanics, Berkeley, CA, USA, 1962, pp. 1075-1085.

[57] Shrive, N. G., Huizier, A. "Letter to the Editor", The Masonry Society Journal, 9(2), 1991.

[58] Shrive, N. G., Reda Taha, M. M. "Effects of creep on new masonry structures", Binda, L. (ed.) Learning from Failure: Long-term Behaviour of Heavy Masonry Structures, WIT Press, Southampton, UK, 2007, pp. 83-108.

[59] Sulem, J., Mühlhaus, H. B. "A continuum model for periodic two-dimensional block structures", Mechanics of Cohesive-frictional Materials, 2(1), pp. 31-46, 1997.

https://doi.org/10.1002/(SICI)1099-1484(199701)2:1<31::AIDCFM24>3.0.CO;2-O|
[60] Taliercio, A. "Closed-form expressions for the macroscopic in-plane elastic and creep coefficients of brick masonry", International Journal of Solids and Structures 51(17), pp. 2949-2963, 2014. https://doi.org/10.1016/j.ijsolstr.2014.04.019

[61] Ushaksarei, R., Pietruszczak, S. "Failure Criterion for Structural Masonry Based on Critical Plane Approach", Journal of Engineering Mechanics, 128(7), pp. 769-778, 2002. https://doi.org/10.1061/(ASCE)0733-9399(2002)128:7(769)

[62] Zucchini, A., Lourenço, P. B. "A coupled homogenization-damage model for masonry cracking", Computers and Structures, 82(1112), pp. 917-929, 2004.

https://doi.org/10.1016/j.compstruc.2004.02.020 